\title{
Balancing Herbal Medicine and Functional Food for Prevention and Treatment of Cardiometabolic Diseases through Modulating Gut Microbiota
}

OPEN ACCESS

Edited by:

Suhelen Egan,

University of New South Wales,

Australia

Reviewed by:

Douglas Morrison

University of Glasgow,

United Kingdom

Rohit Gundamaraju,

University of Tasmania, Australia

*Correspondence: Yan Zhu

yanzhu.harvard@iCloud.com

Specialty section:

This article was submitted to

Microbial Symbioses,

a section of the journal

Frontiers in Microbiology

Received: 30 March 2017

Accepted: 19 October 2017

Published: 08 November 2017

Citation:

Lyu M, Wang Y, Fan G, Wang X, Xu S and Zhu Y (2017) Balancing Herbal Medicine and Functional Food for

Prevention and Treatment of

Cardiometabolic Diseases through

Modulating Gut Microbiota.

Front. Microbiol. 8:2146.

doi: 10.3389/fmicb.2017.02146

\begin{abstract}
Ming Lyu ${ }^{1,2}$, Yue-fei Wang ${ }^{1,2}$, Guan-wei Fan ${ }^{1,2,3}$, Xiao-ying Wang ${ }^{1,4}$, Shuang-yong $X \mathrm{u}^{5}$ and Yan Zhu ${ }^{1,2 *}$

${ }^{1}$ Tianjin State Key Laboratory of Modern Chinese Medicine, Tianjin University of Traditional Chinese Medicine, Tianjin, China, ${ }^{2}$ Research and Development Center of TCM, Tianjin International Joint Academy of Biotechnology \& Medicine, Tianjin, China, ${ }^{3}$ Medical Experiment Center, First Teaching Hospital of Tianjin University of Traditional Chinese Medicine, Tianjin, China, ${ }^{4}$ Neuroscience Program, Neuroprotection Research Laboratory, Department of Neurology and Radiology, Massachusetts General Hospital, Harvard Medical School, Boston, MA, United States, ${ }^{5}$ New England Biolabs, Inc., Ipswich, MA, United States
\end{abstract}

It has become apparent that gut microbiota is closely associated with cardiometabolic diseases (CMDs), and alteration in microbiome compositions is also linked to the host environment. Next generation sequencing (NGS) has facilitated in-depth studies on the effects of herbal medicine and functional food on gut microbiota. Both herbal medicine and functional food contain fiber, polyphenols and polysaccharides, exerting prebiotics-like activities in the prevention and treatment of CMDs. The administrations of herbal medicine and functional food lead to increased the abundance of phylum Bacteroidetes, and genus Akkermansia, Bifidobacteria, Lactobacillus, Bacteroides and Prevotella, while reducing phylum Firmicutes and Firmicutes/Bacteroidetes ratio in gut. Both herbal medicine and functional food interact with gut microbiome and alter the microbial metabolites including short-chain fatty acids (SCFAs), bile acids (BAs) and lipopolysaccharides (LPS), which are now correlated with metabolic diseases such as type 2 diabetes (T2D), obesity and non-alcoholic fatty liver disease (NAFLD). In addition, trimethylamine (TMA)-N-oxide (TMAO) is recently linked to atherosclerosis (AS) and cardiovascular disease (CVD) risks. Moreover, gut-organs axes may serve as the potential strategy for treating CMDs with the intervention of herbal medicine and functional food. In summary, a balance between herbal medicine and functional food rich in fiber, polyphenols and polysaccharides plays a vital role in modulating gut microbiota (phylum Bacteroidetes, Firmicutes and Firmicutes/Bacteroidetes ratio, and genus Akkermansia, Bifidobacteria, Lactobacillus, Bacteroides and Prevotella) through SCFAs, BAs, LPS and TMAO signaling regarding CMDs. Targeting gut-organs axes may serve as a new therapeutic strategy for CMDs by herbal medicine and functional food in the future. This review aims to summarize the balance between herbal medicine and functional food utilized for the prevention and treatment of CMDs through modulating gut microbiota.

Keywords: herbal medicine, functional food, cardiovascular disease, metabolic disease, intestinal microbiota 


\section{INTRODUCTION}

The Human Microbiome Project funded by National Institutes of Health (NIH) (Qin et al., 2010) and Metagenomics of the Human Intestinal Tract (MetaHIT) consortium funded by European Commission (Turnbaugh et al., 2007) have promoted better understanding of the functional properties and healthy composition of gut microbiota. Various microbial communities and their genes (the microbiome) are present in human body, influencing human health and diseases (Human Microbiome Project, 2012). The human gut microbiota contains a diverse array of microorganisms, including bacteria, archaea and fungi that colonize the surfaces of the gastrointestinal (GI) tract; bacteriophage are also in high abundance in GI tract (Savage, 1977). Six bacterial phyla dominate the gut microbiota of healthy adult subjects: Firmicutes, Proteobacteria, Bacteroidetes, Fusobacteria, Actinobacteria, and Verrucomicrobia. The intestine hosts $>10^{14}$ microorganisms with critical physiological roles, and the microbial compositions differ along the digestive tract (Aron-Wisnewsky and Clement, 2016). The large intestine, particularly the colon, harbors a complex and dynamic microbial ecosystem with high densities of living bacteria. These bacteria achieve concentrations of approximately $10^{11}-10^{12}$ cells/g of luminal contents (Simon and Gorbach, 1984; Guarner and Malagelada, 2003).

A multitude of literature supports the role of gut microbiota in the development and progression of cardiometabolic diseases (CMDs). CMDs have become a worldwide epidemic, with dramatically increasing prevalence of cardiovascular disease (CVD), obesity, type 2 diabetes (T2D), non-alcoholic fatty liver disease (NAFLD), atherosclerosis (AS), hypertension, and dyslipidemia (Hansen et al., 2015; Aron-Wisnewsky and Clement, 2016; Meyer and Bennett, 2016; Woting and Blaut, 2016; Micha et al., 2017). In the search for novel therapeutic leads, the association of gut microbiota and microbial metabolites with the development of CMDs holds the potential in future drug discovery (Koopen et al., 2016).

Disruption of microbial ecosystems during crucial developmental periods could affect body physiology or cause undesired negative effects. For instance, the overuse of antibiotics in early life is associated with obesity in both humans and rodents (Cho et al., 2012). Herbal medicine such as traditional Chinese medicine (TCM) can be used as an alternative strategy to modulate microbiota and for modern drug discovery. Moreover, certain food components provided benefits beyond basic nutrition, leading to the concept of functional

Abbreviations: CMDs, Cardiometabolic diseases; CVD, cardiovascular disease; T2D, type 2 diabetes; NAFLD, non-alcoholic fatty liver disease; AS, atherosclerosis; CKD, chronic kidney disease; MI, myocardial infarction; SCFAs, short-chain fatty acids; Bas, bile acids; LPS, lipopolysaccharide; TMAO, trimethylamine$\mathrm{N}$-oxide; TCM, traditional Chinese medicine; MetaHIT, Metagenomics of the Human Intestinal Tract; GI, gastrointestinal; HFD, high-fat-diet; UC, ulcerative colitis; AMR, antimicrobial resistance; RSV, resveratrol; GQD, GegenQinlian Decoction; FMO3, flavin-containing monooxygenase 3; GLP-1, glucagon-like peptide-1; MCP1, monocyte chemoattractant protein 1; ZO-1, zona occludens1; FXR, farnesoid X receptor; FGF15, fibroblast growth factor 15; Fiaf, fastinginduced adipose factor; TLR4, toll-like receptor 4; GPAT1, glycerol-3-phosphate acyltransferase 1 . food and nutraceuticals. Functional food offer benefits beyond basic nutrition when consumed regularly as part of a diet. Herbal medicine and functional food produce a large diversity of secondary metabolites which display a broad array of biological and pharmacological properties (Wink, 2015) and are widely accepted as high-efficiency and low toxicity "medicinal diets" which are capable of avoiding certain side-effects. In this review article, recently discovered mechanisms of herbal medicine and functional food are summarized and their contributions to prevention and treatment of CMDs through modulating microbiota are also outlined.

\section{GUT MICROBIOTA IN CMDS}

\section{Gut Microbiota in Cardiovascular Diseases}

In recent years, an increasing number of reseachers begin to pay their attention to the mutual effects on intestinal flora and CVDs, resulting from the new findings of gut microbederived metabolite trimethylamine (TMA)-N-oxide (TMAO). Gut microbiota has an intimate relationship with CVDs, including thrombosis, AS, myocardial infarction (MI) and stroke.

\section{TMAO}

TMAO was first identified as a contributor to CVD in a large clinical cohort of 1,876 subjects by Stanley L. Hazen team using an untargeted metabolomics platform (Wang et al., 2011). In a subsequent expansion study of 4,007 subjects undergoing elective coronary angiography indicated an association between elevated TMAO levels in plasma and increased risk for major adverse cardiovascular events (MACE) over a 3-year period in humans (Tang et al., 2013). Then, increasing clinical reports support an involvement of plasma TMAO levels in the etiology of various CVDs. For example, elevated plasma TMAO levels in patients predict a high atherosclerotic burden (Senthong et al., 2016a), long-term adverse event risk and incremental prognostic of peripheral artery disease (PAD) (Senthong et al., 2016b), higher long-term mortality risk of coronary artery disease (CAD) (Senthong et al., 2016c), and adverse clinical outcomes in heart failure (HF) (Tang et al., 2014, 2015b; Troseid et al., 2015). Higher TMAO levels provide clinical utility in risk stratification of acute coronary syndromes (ACS) (Li X. S. et al., 2017) showing a direct pro-thrombotic effect (Zhu et al., 2017), predict close association with poor prognosis of MI (Suzuki et al., 2017). A systematic review and meta-analysis reconfirmed elevated concentrations of TMAO and its precursor TMA were associated with increased risks of MACE (Heianza et al., 2017). Furthermore, gut microbiota played an obligatory role in the metabolism of TMA, eight species (Anaerococcus hydrogenalis, Clostridium asparagiforme, Clostridium hathewayi, Clostridium sporogenes, Escherichia fergusonii, Proteus penneri, Providenciarett geri, and Edwardsiella tarda) in two different phyla (Firmicutes and Proteobacteria) and six genera correlated with choline consumption and TMA accumulation were identified (Romano et al., 2015). Undoubtedly, TMAO had become a new biomarker in diagnosis of CVD.

The conclusions of cumulative reports on the metaorganismal metabolic pathway for TMAO production and 
its possible mechanisms resulting in CVD are highlighted: (1)TMA production: phosphatidylcholine (PC), choline, and Lcarnitine, generating the precursor TMA by gut microbiota cleavage, were abundant in dietary foods such as red meat, shellfish, egg yolk and high-fat dairy products (Wang et al., 2011). Until now, either choline or L-carnitine as substrate, TMA is produced by two identified distinct microbial enzyme systems. Catalytic unit (cutC) and a regulatory polypeptide (cutD) are required for TMA production from choline (Craciun and Balskus, 2012; Craciun et al., 2014). The catalytic protein $(C n t A)$ and the regulatory protein $(C n t B)$ are involved in TMA production form L-carnitine (Zhu et al., 2014). (2TMA $\rightarrow$ TMAO: hepatic flavin monooxygenase 3 (FMO3) expression was up-regulated by bile acids (BAs) via nuclear receptor farnesoid X receptor (FXR) activation (Bennett et al., 2013). TMA was readily absorbed and traveled through the portal circulation to the liver and was oxidized into TMAO by FMO3 (Wang et al., 2011; Bennett et al., 2013). (3TMAO induced or enhanced cell phenotypic changes: elevated plasma TMAO induced endothelial dysfunction via activating reactive oxygen species (ROS)/thioredoxin-interactive protein (TXNIP)/nodlikereceptor family pyrin domain containing 3 (NLRP3) inflammasome (Sun X. et al., 2016) and impairing endothelial nitric oxide synthase (eNOS)-derived NO bioavailability ( $\mathrm{Hu}$ et al., 2015; Li T. et al., 2017). In addition, TMAO accelerated vascular inflammation through mitogen-activated protein kinase

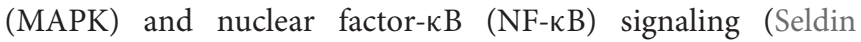
et al., 2016), reduced endothelial self-repair, and increased monocyte adhesion partly via the pathway of protein kinase C (PKC)/NF-кB/vascular cell adhesion molecule-1 (VCAM-1) activation (Ma et al., 2017). Moreover, TMAO contributed to macrophage cholesterol accumulation and foam cell formation (Wang et al., 2011). Furthermore, TMAO elevated platelet hyperreactivity, enhancing agonists-induced platelet activation through intracellular $\mathrm{Ca}^{2+}$ mobilization (Zhu et al., 2016). These changes in cell phenotype contribute to atherosclerotic CVD. (4)TMAO promoted CAD in animal studies: TMAO accelerated AS by reversing cholesterol transport and altering bile acids (BAs) composition (Koeth et al., 2013), enhanced thrombosis formation by activating platelet (Zhu et al., 2016), exacerbated pressure overload-induced heart failure by inducting adverse cardiac remodeling (Organ et al., 2016). Attention should be paid to the gender identity in the study of TMAO synthesis, since FMO3 expression is higher in females than males in both human and mouse (Bennett et al., 2013).

For further research, pharmacologic inhibition of TMAO producition will be a potential therapeutic strategy to reduce CVD events by targeting microbial community, microbial enzyme and/or FMO3 expression. The mechanisms of TMAO pathway (PC, choline, L-carnitine $\rightarrow$ TMA $\rightarrow$ FMO3 $\rightarrow$ TMAO) linking the specific microbiota to cardiovascular functionis are very important and need to be further elucidated. In addition to the new finding of TMAO, other microbial metobolites such as SCFAs (Marques et al., 2017), BAs (Mayerhofer et al., 2017), and LPS (Pastori et al., 2017) which are beneficial for CVD will not be discussed in details here.

\section{Gut Microbiota in Metabolic Diseases}

A considerable number of publications have reported correlations between gut microbiota and metabolic diseases (Moreno-Indias et al., 2014; Janssen and Kersten, 2015; Greenhill, 2016; Saad et al., 2016; Sonnenburg and Backhed, 2016; Woting and Blaut, 2016). Specific metabolic abnormalities such as pro-inflammatory states, insulin resistance, glucose intolerance, dyslipidemia, high blood pressure and NAFLD, which accompanies gut microbiota dysbiosis, often develop in obese people. Moreover, obesity and T2D are considered as a medical condition, which not only contributes to the risk of developing CVD and cancer, but also negatively affects longevity and quality of life. Here, we describe several crucial mechanisms (mainly about SCFAs, LPS and BAs) that contribute to understanding the correlation between gut microbiota and obestiy/T2D.

\section{SCFAs}

The intestinal microbial fermentation and degradation of dietary nondigestible fiber and polysaccharides to SCFAs (acetate, propionate and butyrate) are regarded as potential metabolic targets to prevent obesity/T2D in glucose metabolism and insulin resistance. Besides, several mechanisms correlate with SCFAs affect body weight via energy intake and energy harvesting, and link with insulin sensitivity through inflammatory resoponse, lipid storage and adipose tissue function (Canfora et al., 2015). SCFAs, serving as energy substrates, directly inhibit histone deacetylases (HDACs) and activate G-protein-coupled recepotors (GPCRs). Moreover, butyrate also has effect on epithelial barrier function by increasing mucus production and protein zonula occludens-1 (ZO)-1, occludin expression (Bordin et al., 2004; Peng et al., 2007). GPR41and GPR43 targets are of significance for SCFAs. Gut microbiota promotes adiposity and body weight via SCFAs receptor GPR41. The expression of peptide YY (or PYY), a key hormone involved in the elevation of intestinal transit rate and reduction in energy hearvest, is decreased in GPR41-/- mice (Samuel et al., 2008). On the contrary, SCFAs may prevent obesity via activation of GPR43. Normal diet GPR43 ${ }^{-/-}$mice are obese, whereas HFD-fed GPR $43^{+/+}$mice remain lean. Insulin signaling in adipocytes and fat accumulation in white adipose tissue (WAT) are inhibited via acetate-medatied GPR43 (Kimura et al., 2013). These distinct differences remain to be analyzed in how the gut microbiota is modulated. Besides, GPCR43 activation by SCFAs promotes the release of glucagon-like peptide-1 (GPL-1) by intestinal enteroendocrine L cells, thereby leading to insulin release and stimulating glucose tolerance (Tolhurst et al., 2012). Furthermore, a recent paper reported that acetate contributes to GPR43-mediated intestinal IgA response to microbiota, leading to crucial role in intestinal homeostasis maintenance and intestines inflammation denfence (Wu et al., 2016). Apparently, SCFAs-mediated GPCRs signaling in mice shows extensive effects on obesity/T2D, but the role of GPR41/43 signaling in humans remains to be established.

\section{LPS}

Endotoxin LPS is a major component of the gram-negative bacterial (such as Escherichia coli) outer membrane. 
HFD-induced gut microbiota dysbiosis can alter gut permeability and then increase cirlulating LPS levels which promotes lowgrade inflammation and insulin resistance and, ultimately, obesity and T2D in rodents and humans (Cani et al., 2007, 2008; Creely et al., 2007). In addition, Increased intestinal epithelial barrier permeability is due to increased endocannabinoid system tone (Muccioli et al., 2010) and tight junctions (ZO)-1, occludin and claudin-1 expression (Wang J. H. et al., 2014). LPS stimulates inflammatory response mainly by binding to CD14/Toll-like receptor 4 (TLR4) which is respnsible for the recruitment and activation of MyD88 adaptor and NF- $\mathrm{B}$ transcription factor, inducing the pro-inflammatory factors interleukin-6 (IL-6), interleukin-1 $\beta$ (IL-1 $\beta$ ) and monocyte chemoattractant protein-1 (MCP-1) secretion (Hennessy et al., 2010), and therefore it triggers matabolic diseases (Robbins et al., 2014; Kang et al., 2016). Metabolic characteristics of obesity and T2D in mice were not initiated by injecting LPS when CD14/TLR4 receptor was genetically deleted, showing the significant contribution of LPS/CD14/TLR4 signaling (Shi et al., 2006; Cani et al., 2007, 2008; Poggi et al., 2007). Unexpectedly, insulin is more sensitive in TLR4 ${ }^{-1-}$ (Shi et al., 2006) mice, but less in TLR6 ${ }^{-/-}$(VijayKumar et al., 2010) mice with the modulator of gut microbiota than wild-type controls.

\section{BAs}

BAs is produced in the liver from cholesterol and metabolized in the gut by the intestinal microbiota (Midtvedt, 1974). Inversely, BAs can modulate gut microbial composition via innate immune genes activation in the small intestine (Wahlstrom et al., 2016). Cholic acid (CA) and chenodeoxycholic acid (CDCA) are the primary BAs produced in humans, whereas $\mathrm{CA}$ and muricholic acids (MCAs) are generated in rodents. Besides, mice also produce ursodeoxycholic acid (UDCA) as primary BAs (Sayin et al., 2013), whereas as a secondary BA in human (Ishizaki et al., 2005). The primary BAs are converted into secondary BAs by gut microbial modifications. BAs play multiple roles in the control of obesity/T2D related glucose and lipid metabolism, and energy homeostasis by activating the nuclear FXR and the cytoplasmic G protein-coupled membrane receptor 5 (TGR5) which regulate a large number metabolic pathways in the host (Thomas et al., 2008, 2009; Wahlstrom et al., 2016). On one hand, FXR is activated mainly by the CA and CDCA (Makishima et al., 1999; Parks et al., 1999; Wang et al., 1999), while TGR5 is stimulated mostly by LCA and DCA which are secondary BAs metabolized from CA and CDCA (Maruyama et al., 2002; Chen X. et al., 2011). On another, T $\alpha / \beta M C A$ (primary BAs in mice) and UDCA inhibit FXR activation (Li et al., 2013; Sayin et al., 2013; Mueller et al., 2015). Furthermore, GLP-1 synthesis is inhibited by FXR activition (Trabelsi et al., 2015), while it is activated and secreted by TGR 5 activation in colonic L cells (Thomas et al., 2009). GLP-1 signaling may be exploited into a new therapy for T2D with the help of gut microbiota (Claus, 2017; Grasset et al., 2017). Hepatic cholesterol 7a-hydroxylase (CYP7A1) is regulated by intestinal FXR with the contribution of a fibroblast growth factor15 (FGF15) activity (Inagaki et al., 2005). What's more, recent study showed HFD-fed FXR ${ }^{-/-}$mice an obesity phenotype compared to the wild-type mice (Parséus et al., 2016). Thus, targeting BAs, FXR, and/or TGR5 signaling with microbiota, may shed a new light on preventing or treating metabolic diseases. At present, our knowledge on the mutual effects between BAs and gut microbiota is still far from complete.

In recent studies, the gut metabolite TMAO is also found to have an intimate relationship with metabolic diseases such as T2D (Dambrova et al., 2016; Tang et al., 2016; Schugar et al., 2017), NAFLD (Chen Y. M. et al., 2016), chronic kidney disease (CKD) (Tang et al., 2015a; Xu K. Y. et al., 2017) and bariatric surgery (Troseid et al., 2016), along with the metabolic functions including insulin resistance (Oellgaard et al., 2017) and BAs metabolism (Wilson et al., 2016). With the rapid development in the field of intestinal microbiota, the gut metabolites like TMAO, SCFAs, LPS, and BAs with their signaling interplay between microbiota, have evolved as promising avenues for prevention and treatment of CMDs. Herbal medicine and functionl food with the properity of muti-ingredient, mutitarget and muti-pathway action may serve as a prebiotic-like remediation (Laparra and Sanz, 2010; Xu J. et al., 2017). How might they work in CMDs by modulating gut microbiota are discussed below.

\section{HERBAL MEDICINE AND GUT MICROBIOTA}

The effectiveness of antibiotics in modern medicine has diminished somewhat due to the development of multi-drug resistant bacteria after using for more than 70 years. New classes of antimicrobial drugs are unlikely to become widely available any time soon (Laxminarayan et al., 2016). If and when they do, bacteria, viruses and other microbes will again evolve antimicrobial resistance (AMR) through variety of ways including horizontal gene transfer of mobile genetic elements (Carroll et al., 2014; Jorgensen et al., 2016). Experimental evidence, particularly rodent studies, showed convincingly that prebiotics, non-digestible, fermentable carbohydrates and fibers are capable of enhancing the growth of specific beneficial gut bacteria, thus reducing body weight, reversing insulin resistance and exerting anti-inflammatory effects (Bindels et al., 2015; Sonnenburg and Backhed, 2016). However, these effects have yet to be confirmed by intervention studies in human. Recent investigations support the idea of the involvement of intestinal bacteria in host metabolism and preventative therapeutic potential of prebiotic interventions for CMDs. Herbal medicine may therefore serve as a potential prebiotic remedy to treat CMDs and complications.

Several herbal medicine formulae, herbals and nutraceuticals that contain fiber, polyphenol, polysaccharide and certain other substances have anti-obese, anti-diabetic and anti-atherosclerotic effects through the modulation of diverse gut microbiota. These herbals with their components have the potential to be a new source for CMD drugs discovery that target specifically the gut microbiota, as summarized in Tables 1-3. According to the early direct evidence in 187 T2D patients, a herbal formula Gegen Qinlian Decoction (GQD) including four herbs: Gegen (Radix Puerariae), Huangqin (Radix Scutellariae), Huanglian 


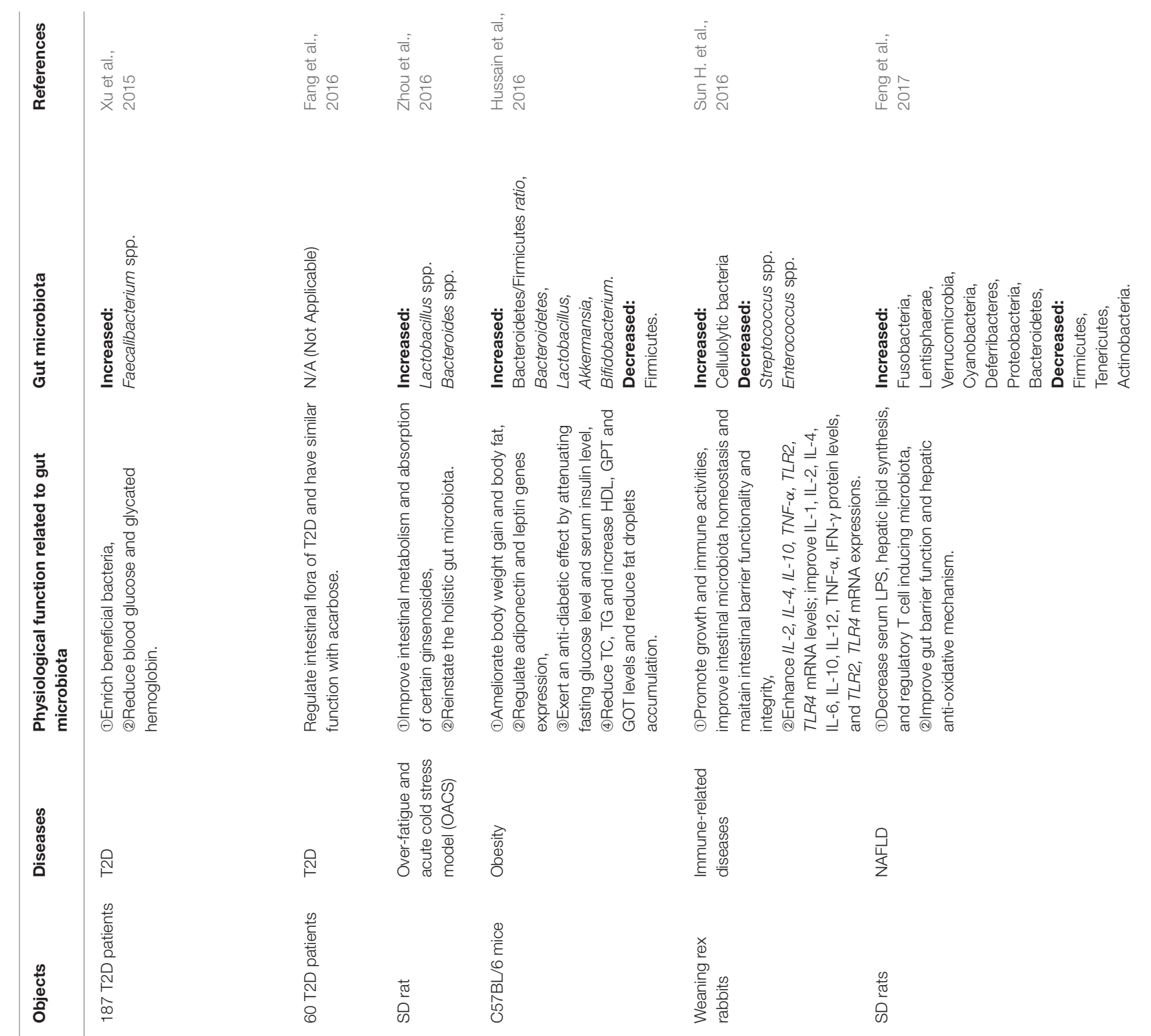



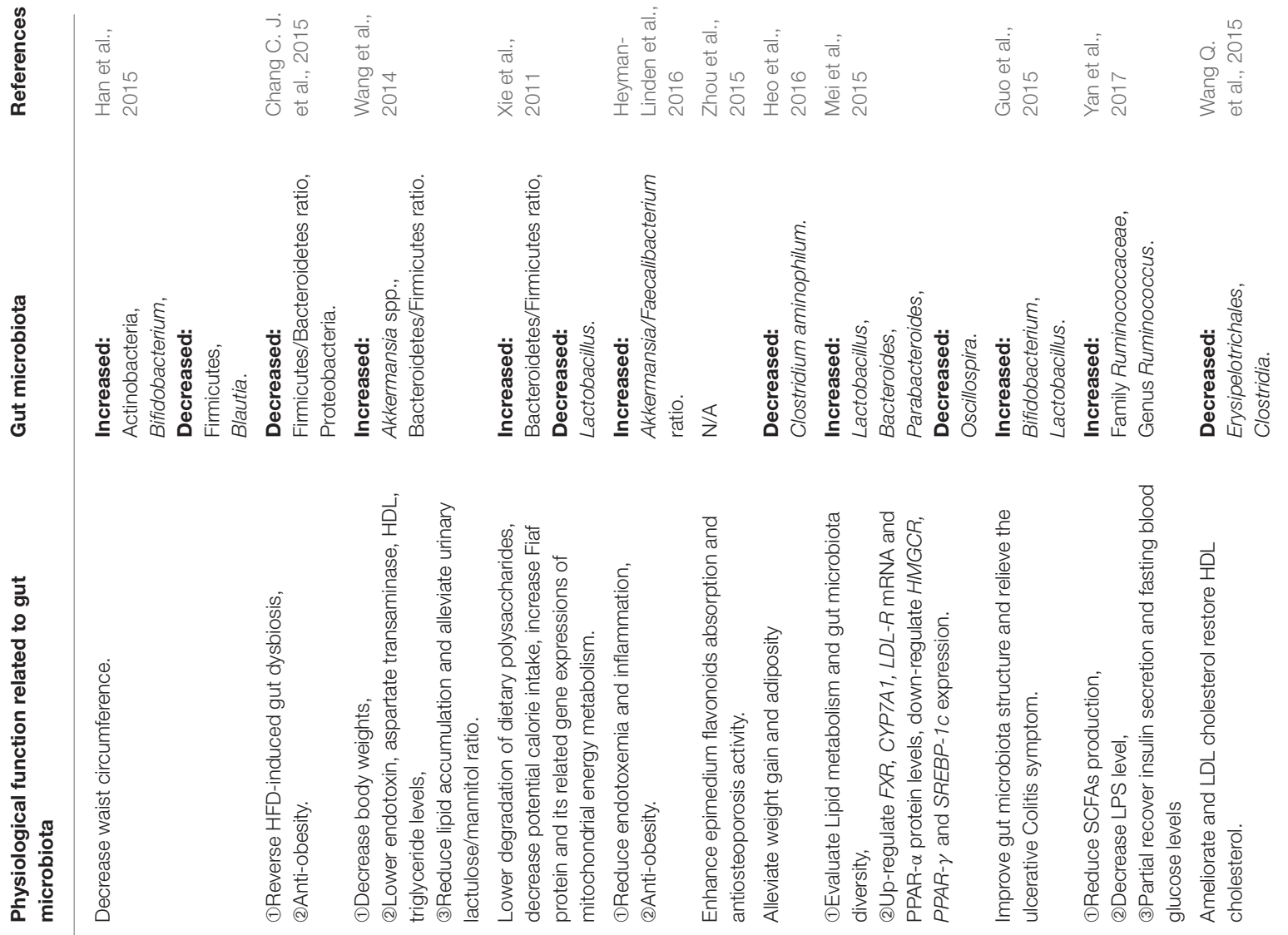

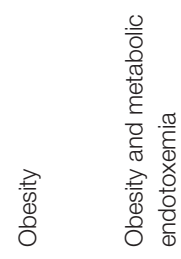
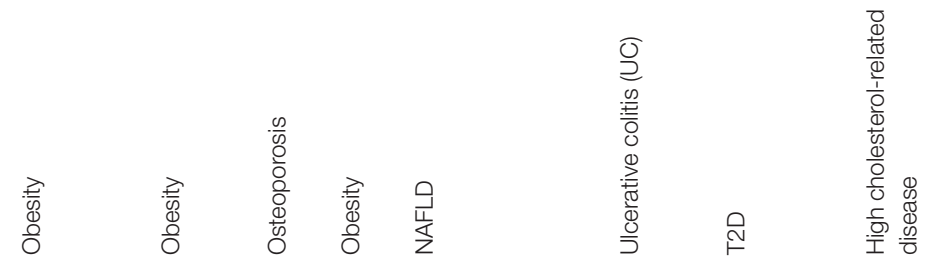

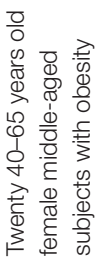
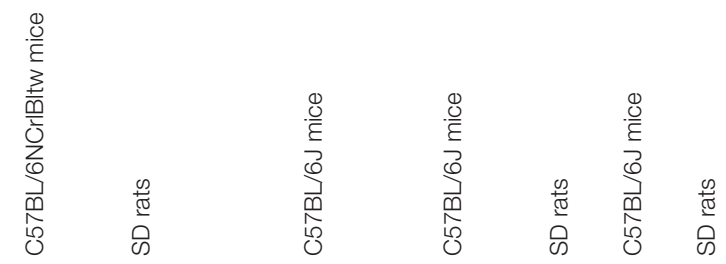

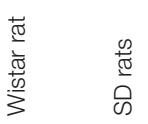
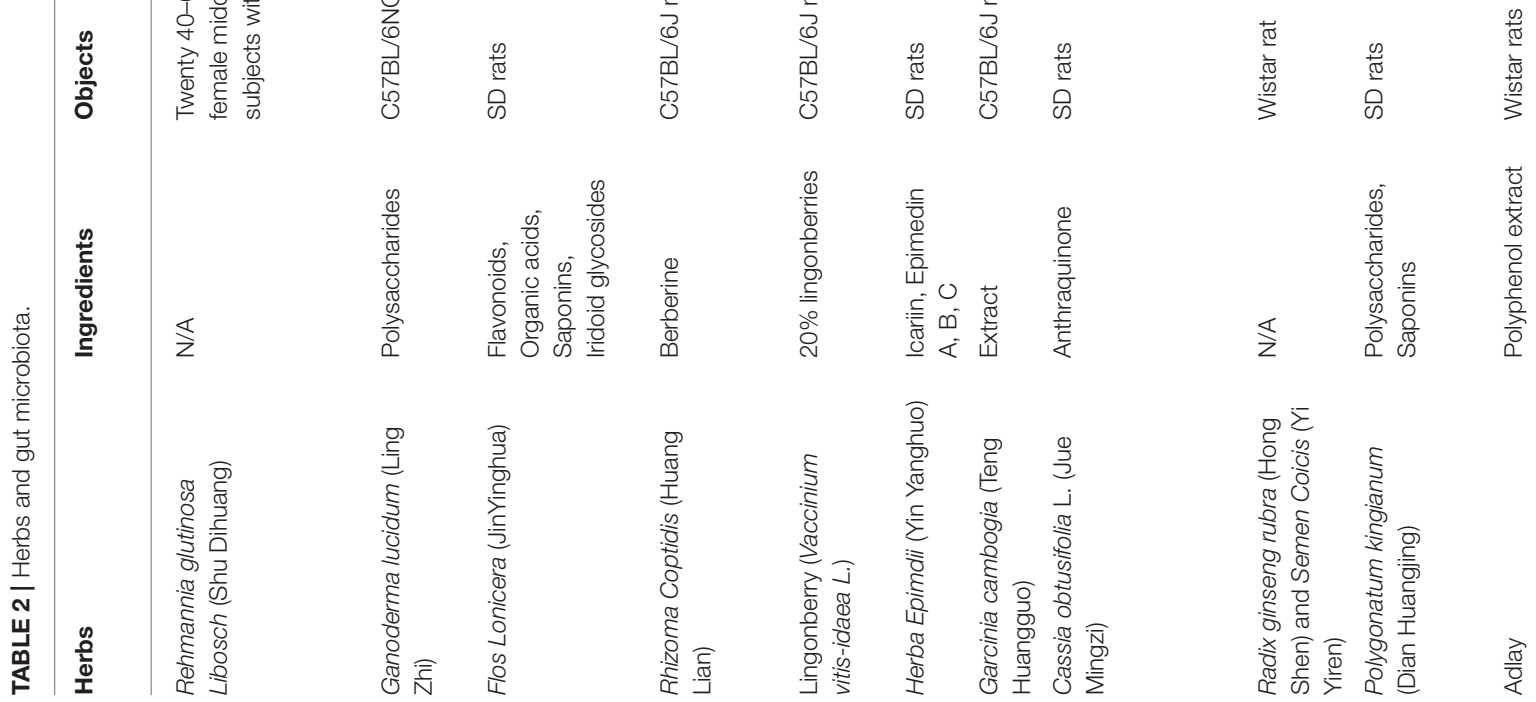


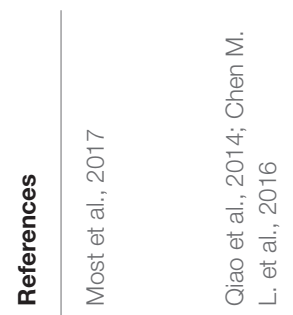

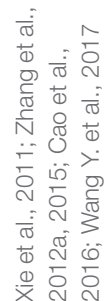

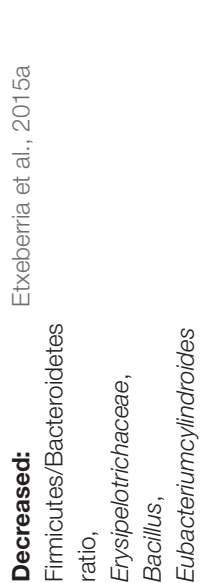

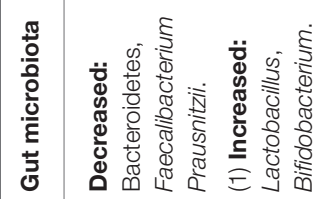

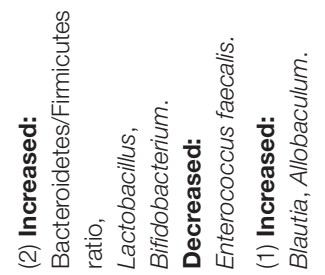

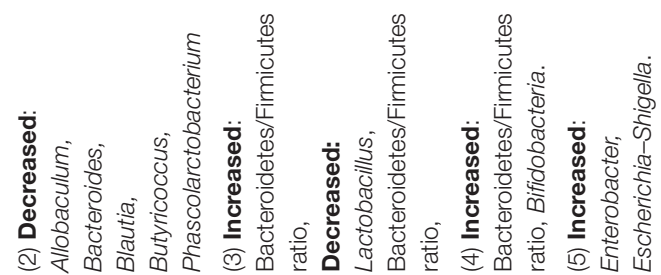

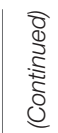
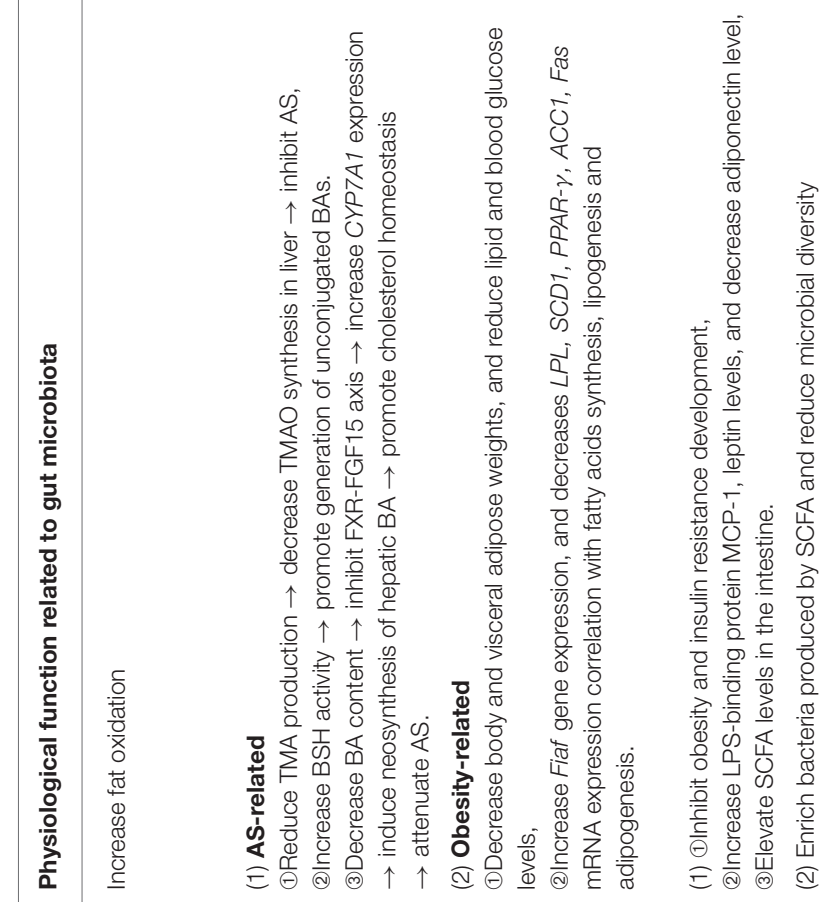

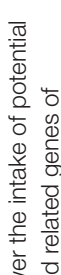

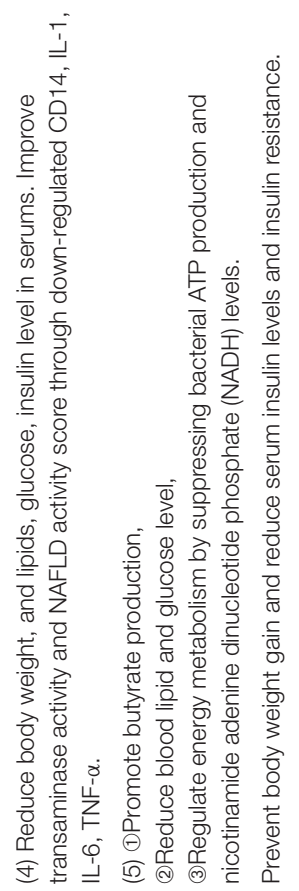

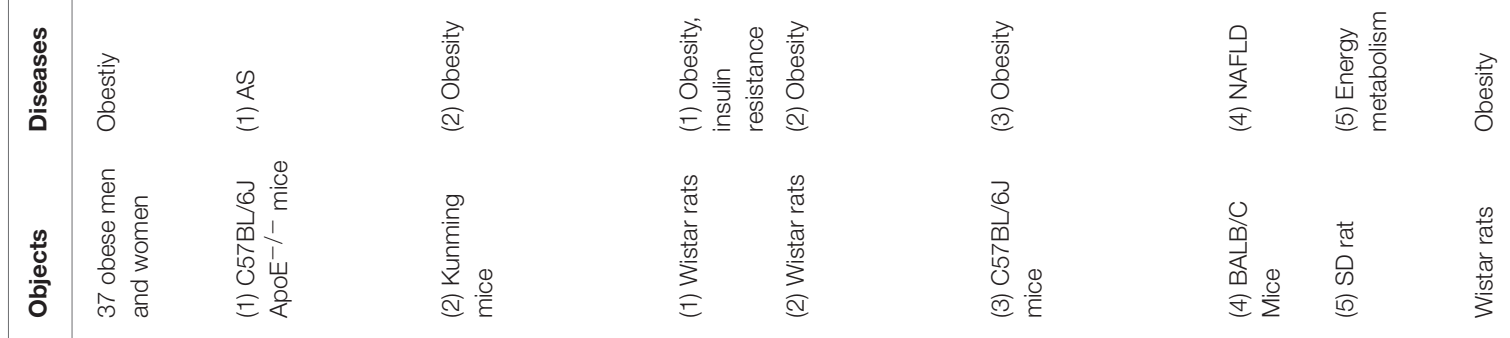

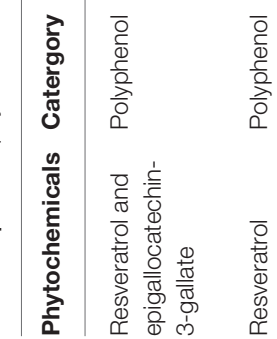

$\frac{\text { 을 }}{\frac{0}{0.0}}$

$\overline{0}$
$\frac{0}{\overline{0}}$
o.
흥
0

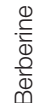

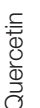




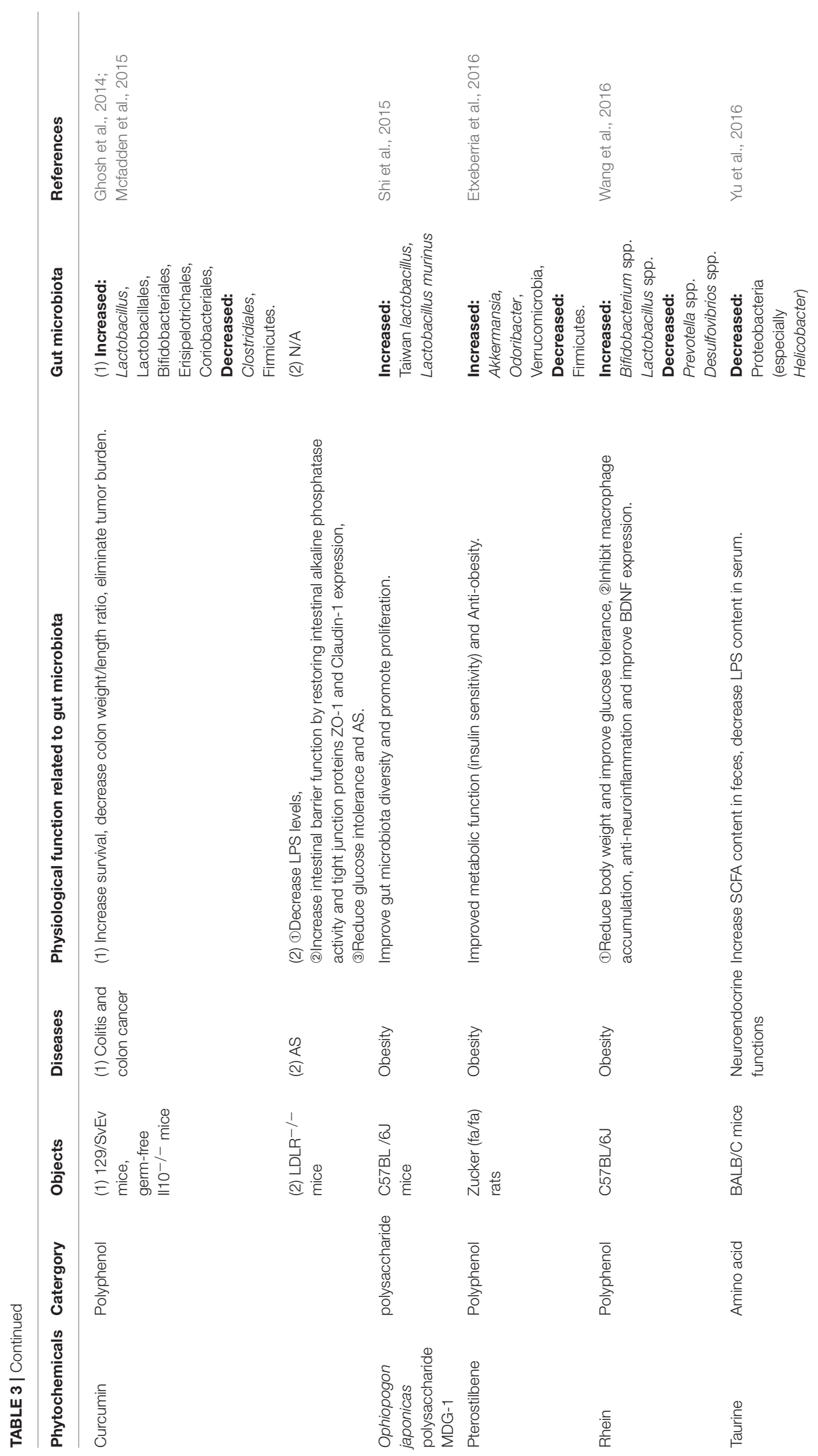


(RhizomaCoptidis) and Gancao (Honey-fried Licorice Root), showed the anti-T2D effect partly by enriching the amounts of specific beneficial bacteria Faecalibacterium spp. (Xu et al., 2015). Interestingly, both Rhizoma coptidis (as the major component of GQD) and berberine (as the main phytochemicals of Rhizoma coptidis) are confimed to have an anti-obese effect by inhibiting the ratio of Firmicutes/Bacteroidetes, and lowering the growth of Lactobacillus (a classical type of Firmicutes) in HFD-fed mice feces. In addition, Rhizoma coptidis and berberine can reduce HFD-induced body and visecral adipose weights, and blood glucose and lipid levels in mice (Xie et al., 2011).What's more, berberine increases putative SCFA-producing bacteria, including Blautia, Allobaculum, Bacteriodes, Blautia, Butyricoccus, and Phascolarctobacterium, possibly leading to anti-obese and antidiabetic effects in the host (Zhang et al., 2012a, 2015). Rhizoma coptidis and berberine, also the main ingredients of GQD, may contribute to the significant resistance to metabolic disease by targeting intestinal microbiota, which need to be further confirmed in clinical trials. A recent study showed that berberine improved non-alcoholic steatohepatitis (NASH) by restoring Biffidobacteria and reducing Firmicutes/Bacteroidetes ratio (Cao et al., 2016). Another herbal formula Qushi Huayu Decoction (QHD), a mixture of five herbs (Artemisia capillaries Thunb, Gardenia jasminoides Ellis, Fallopia japonica, Curcuma longa L., and Hypericum japonicum Thunb.) and two active ingredients (geniposide and chlorogenic acid) reduces oxidative stress and inflammatory response in liver by inducing glutathione-generating enzymes, decreases lipid synthesis and elevates steatosis by inhibiting glucokinase expression, and ameliorates gut barrier function and alleviates liver inflammation by inducing Treg-producing baceria. In these studies, 12 phyla of gut bacteria were altered, including increased Fusobacteria, Lentisphaerae, Verrucomicrobia, Cyanobacteria, Deferribacteres, Proteobacteria, and Bacteroidetes, as well as decreased Firmicutes, Tenericutes and Actinobacteria (Yang et al., 2017).

Resveratrol (RSV), a natural polyphenolic compound extrated from herbal medicine Rhixoma Polygoni Cuspidati or functional food peanut, grape, and Fructus Mori, exerts antioxidant, antiinflammatory (Walker et al., 2014), anti-tumor, cardioprotective, aging-delay, and anti-obesity effects (Baur and Sinclair, 2006; Zhang et al., 2012b). On one hand, RSV decreases TMAO levels and increases hepatic BA neosynthesis via increasing the genera Lactobacillus and Bifidobacterium, thus attenuating TMAOinduced AS in $\mathrm{ApoE}^{-/-}$mice. RSV-induced BA neosynthesis was partially mediated through the enterohepatic FXR-fibroblast growth factor 15 (FGF15) axis (Chen M. L. et al., 2016). On another, RSV increases the ratio of Bacteroidetes/Firmicutes and the growth of Lactobacillus and Bifidobacterium. It also reduces the growth of Enterococcus faecalis through fastinginduced adipose factor (Fiaf, a key gene expresses in the intestine and negatievely regulated by interstinal flora) signaling, decelerating the development of obesity (Qiao et al., 2014). RSV is probably an unique and firstly reported natural product that mediates protection against both CVD and metabolic diseases via gut microbiota to date. In addition, quercetin, a key member of the polyphenol family, is discovered in numerous medicinal botanicals, including Ginkgo biloba, Hypericum perforatum, and Sambucus canadensi and also found in a variety of functional foods including apple, grape, berry, onion and tea (Li Y. et al., 2016). Intake of quercetin reduced body weight gain and attenuated serum insulin levels by reducing Firmicutes/Bacteroidetes ratio and inhibiting the growth of bacterial species Erysipelotrichaceae, Bacillus and Eubacterium cylindroides, which correlated with HFD-induced obesity (Etxeberria et al., 2015a). Moreover, it was shown in a recent study that curcumin, the major polyphenolic ingredient of an edible herb Curcuma longa L. improved intestinal barrier function by modulation of intracellular signaling, and organization of tight junctions, providing a mechanism that curcumin modulates chronic inflammatory diseases despite poor bioavailability (Wang J. et al., 2017). The details of some other herbal medicines, including formulae, herbals and phytochemicals reportedly to achieve their therapeutic effects for CMDs through gut microbiota modulation are summarized in Tables 1-3.

\section{FUNCTIONAL FOOD AND MICROBIOTA}

Functional food has the advantages of wide availability, ease of preparation and fewer adverse effects. They could be well suited for CMDs remedies due to their potential effects such as anti-inflammatory, antioxidants, antiestrogenics, immunomodulatory, whereas purified active compounds are preferable as pharmaceutical drugs for the treatment of severe chronic symptoms (Martel et al., 2016; Meyer and Bennett, 2016). Epidemiological studies have identified associations between frequent consumption of fruits, vegetables, whole grains and teas, which are rich in fiber, polyphenol, and polysaccharide could reduce the risk of CMDs (Woodside et al., 2013; Klinder et al., 2016). These phytochemicals and their metabolic products may inhibit pathogenic bacteria while stimulating the growth of beneficial bacteria for CMDs (Laparra and Sanz, 2010).

Apples are among the most frequently consumed fruits to prevent obestiy by modulating gut microbiota with their multiple components, including fiber, pectin (Jiang et al., 2016), procyanidins (Masumoto et al., 2016) and polysaccharides (Wang S. et al., 2017). Administration with apple procyanidins (a subclass of polyphenols) for 20 weeks was able to reduce obesity, decrease lipid metabolism related genes expression, lower LPS levels and gut permeabiliby through decreasing the Firmicutes/Bacteroidetes ratio and increasing Akkermansia proportion (Masumoto et al., 2016). In addition, treatment with apple polysaccharide inhibited chronic inflammation, gut permeability, and SCFAs production, leading to lower abundance of Firmicutes and Fusobacteium, and higher Bacteroidetes and Lactobacillus in HFD-fed rats (Wang S. et al., 2017). Furthermore, the reciprocity between apple ingredients and the gut microbioa may benefit cardiovascular health (Koutsos et al., 2015). Unexpectedly, diet apple fiber and flavone were positively associated with Blautia, Lactobacillus, Bifidobacterium, and Faecalibacterium, showing great significance for the patients who suffer from systemic lupus erythematosus (SLE) (Cuervo 
et al., 2015; Table 4). As another example, ingestion of laminarin, a kind of polysaccharides extracted from Laminaria japonica, by HFD-fed mice significantly increased genus Bacteroides and decreased Firmicutes, with elevated energy metabolism (Nguyen et al., 2016; Table 5). Besides, 3,3-dimethyl-1-butanol (DMB), the structural analog of choline detected in some functional food such as balsamic vinegars, red wines, and olive oils (Kitai and Tang, 2017), is an inhibitor of TMA formation through inhibition of microbial TMA lyases. Therefore, it inhibited choline dietpromoted macrophage foam cell formation and atherosclerotic lesion development without altering the circulating cholesterol levels (Wang Z. et al., 2015).

Moreover, vegetables (e.g., bamboo shoot), whole grains (e.g., wheat, barley and oat), and teas (e.g., green tea, oolong tea, black tea and fuzhuan tea), exert positive effects on CMDs through modulating gut microbiota (Table 4). Numerous instances are detailed in Tables 4, 5. Although, intervention studies conducted both in animals and humans have demonstrated beneficial effects of functonal foods on anti-inflammation, vascular function, and energy metabolism, the apparent association with altered gut microbiota is still lacking.

\section{OVERLAPING EFFECTS BETWEEN HERBAL MEDICINE AND FUNCTIONAL FOOD ON GUT MICROBIOTA}

Medicine and food deriving from the same source has been realized since ancient times. In TCM, food is conceptualized according to both nutritional and functional aspects, and can be used to treat illnesses. The "medicine-food homology" concept has given a new meaning since the discovery of human-microbiota existing as a whole symbiotic ecosystem. Interestingly, a series of overlapping characteristics through modulating gut microbiota for CMDs between herbal medicine and functional food are uncovered based on the description above (Tables 1-5): (1)shared components, (2)similar functions, (3)common mechanisms, and (4)same intestinal microbiota.

First and foremost, there is no absolute boundary between medicine and food. Some medicines are food whereas certain foods can be employed as medicine. Lonicera japonica Thunb, Cassia obtusifolia L., Semen Coicis, adlay, Zingiber officinale Roscoe (major ingredient: curcumin), and mulberry (main ingredient: RSV), are edible medicines, there are only dosage differences between edible and medicinal use. These herbs not only belong to medicine with valid efficacies for CMDs remedy, but also are delicious food with rich nutrients. Besides, some medicines have been developed into nutraceuticals, which contain important natural bioactive compounds that confer health-promoting and medical benefits to humans, such as Ganoderma lucidum, Herba Epimdii, Ophiopogon japonicas, Rehmannia glutinosa Libosch, Rheum rhabarbarum (major ingredient: rhein), and lingonberry, see Tables 1-3. Furthermore, many foods could serve as nutraceutical candidates, and some of those, such as pomegranate peel, bamboo shoot, grape (major ingredient: RSV), and laminarin have the potential to branch into medicines (Tables 4, 5).
Secondly, the components of fibers (e.g., bamboo shoot, nopal, and yellow pea), polyphenols (e.g., GQD, Flos Lonicera, adlay, apple, grape, orange, nopal and tea) and/or polysaccharides (e.g., Ginseng decoction, Yupingfeng, Ganoderma lucidum, Polygonatum kingianum, Ophiopogon japonicas, apple and barley) are shared in most herbals and foods, exerting prebioticslike effects for CMDs, which can be seen in Tables 1-5. Moreover, polyphenol phytochemicals such as RSV and quercetin are present in both herbals and foods. These components are able to escape absorption in the upper gastrointestinal tract and reach the large intestine without breaking down. Thus, these components can also be converted by local microbiota to biologically active and bioavailable metabolites with systemic effects.

Thirdly, just as what we have introduced above, gut microbiota-derived metabolites such as SCFAs, LPS, Bas, and TMAO are the most likely microbial metabolites linking CMDs remedy to intestinal microbiota. Numerous herbals and foods are likely to prevent and treat CMDs through these mediators. Improved gut permeability and gut integrity in conjunction with the increased expression of ZO-1 and/or occludin-1 and/or claudin-1, resulted in reduction of circulating LPS levels and a series of inflammatory response, which are affected by herbals (Yupingfeng polysaccharides enhanced immunity, Sun H. et al., 2016). QHD was used in the treatment of NAFLD (Feng et al., 2017); Flos Lonicera ameliorated obesity (Wang J. H. et al., 2014); curcumin attenuated AS (Ghosh et al., 2014) and foods [apple derived polyphenols and polysaccharide prevented obesity, (Masumoto et al., 2016; Wang S. et al., 2017), nopal and capsaicin were used in combacting obesity (Kang et al., 2017; Sanchez-Tapia et al., 2017)]. SCFAs production was shown to restore aberrant levels of gut hormones such as GLP-1, PYY, and the activation of GPR43. SCFAs production are also promoted by berberine in energy metabolism, insulin resistance and obesity (Xie et al., 2011; Zhang et al., 2015; Xu J. H. et al., 2017); elevated by apple polysaccharide in chronic inflammation, and enriched by oat in obesity treatment (Wang S. et al., 2017). In addition, Polygonatum kingianum and taurine intervene with both SCFAs and LPS levels in different CMDs (Yu et al., 2016; Yan et al., 2017). TMAO levels were inhibited via the reduction of TMA formation by RSV and then attenuate AS (Wang Z. et al., 2015; Chen M. L. et al., 2016). At the same time, RSV increased BAs deconjugation and fecal excretion by enhancing the activity of hydrolase activity, which displayed correlation with the lowered BA content in ilealby suppressing FXR-FGF15 axis and promoting CYP7A1 expression (Chen M. L. et al., 2016). All of these interventions are along with the changed microbiota composition. An increasing number of metabolic pathway and potential mechanisms are studied on the mediators of SCFAs, LPS, BAs and TMAO. These studies provide a better understanding of how herbals and foods prevent or treat CMDs by gut microbiota. The cross-talk between these mediators and specific alteration of intestinal bacteria in host physiology, as well as the precise contributing elements in herbals and foods for CMDs remedy shoud be subjects for future studies.

Finally, previous work has established that genera Clostridium, Lactobacillus and Ruminococcus, as well as the butyrate producers 


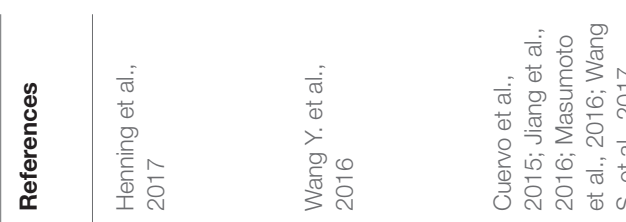

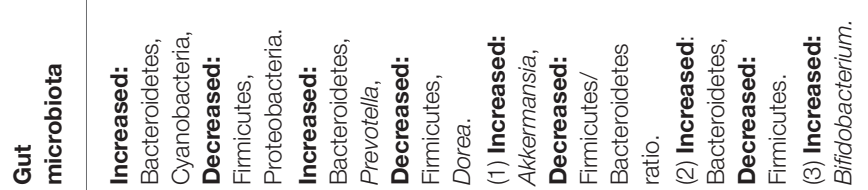

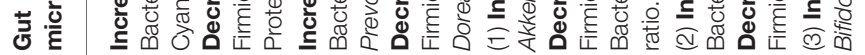

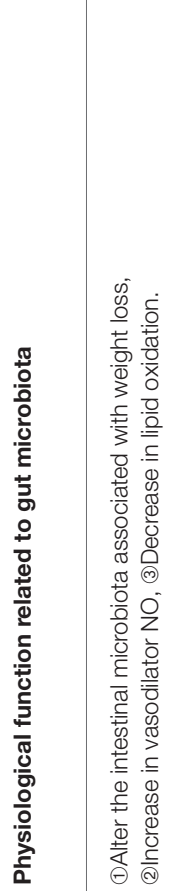

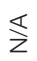

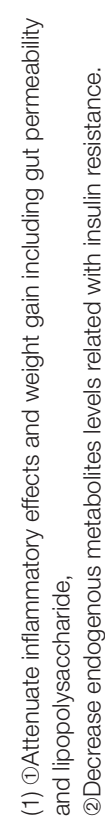

$\frac{3}{0}$
0
0
0
0
0

$\stackrel{0}{\stackrel{0}{\varepsilon}}$
$\stackrel{0}{0}$
$\stackrel{0}{0}$
0
0
0
0

๘ึ

$\frac{\circ}{\circ} \frac{\circ}{\bar{\varepsilon}}$

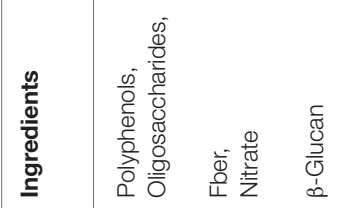

竞

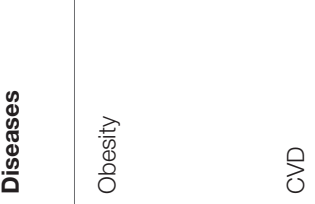$$
\text { 告 }
$$

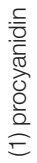

$\frac{\infty}{\frac{0}{2}}$

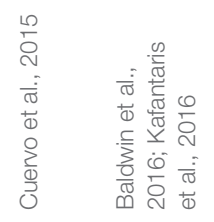
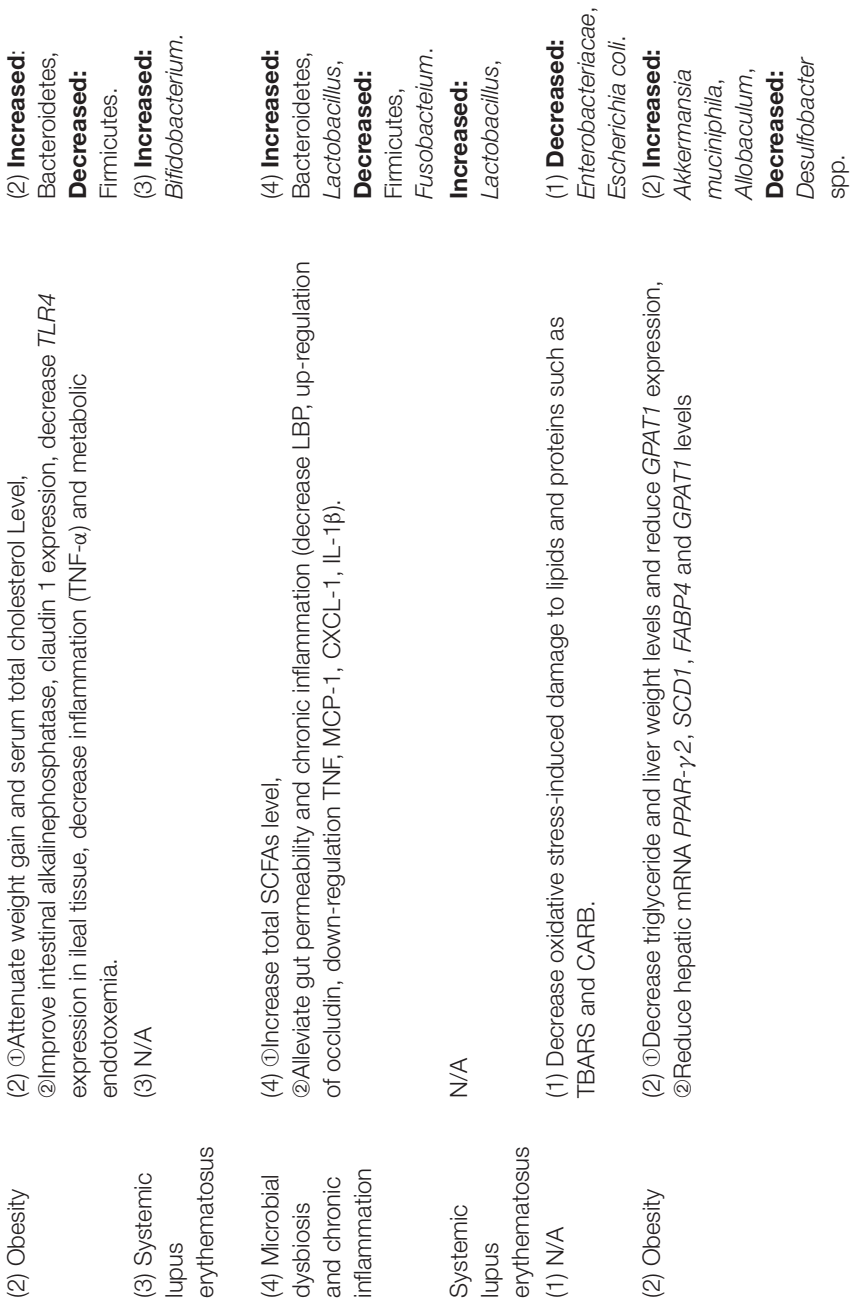

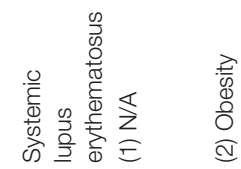

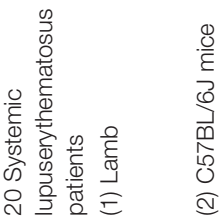
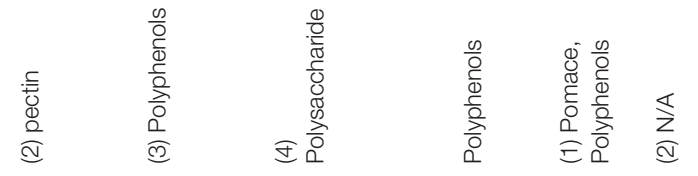

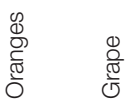




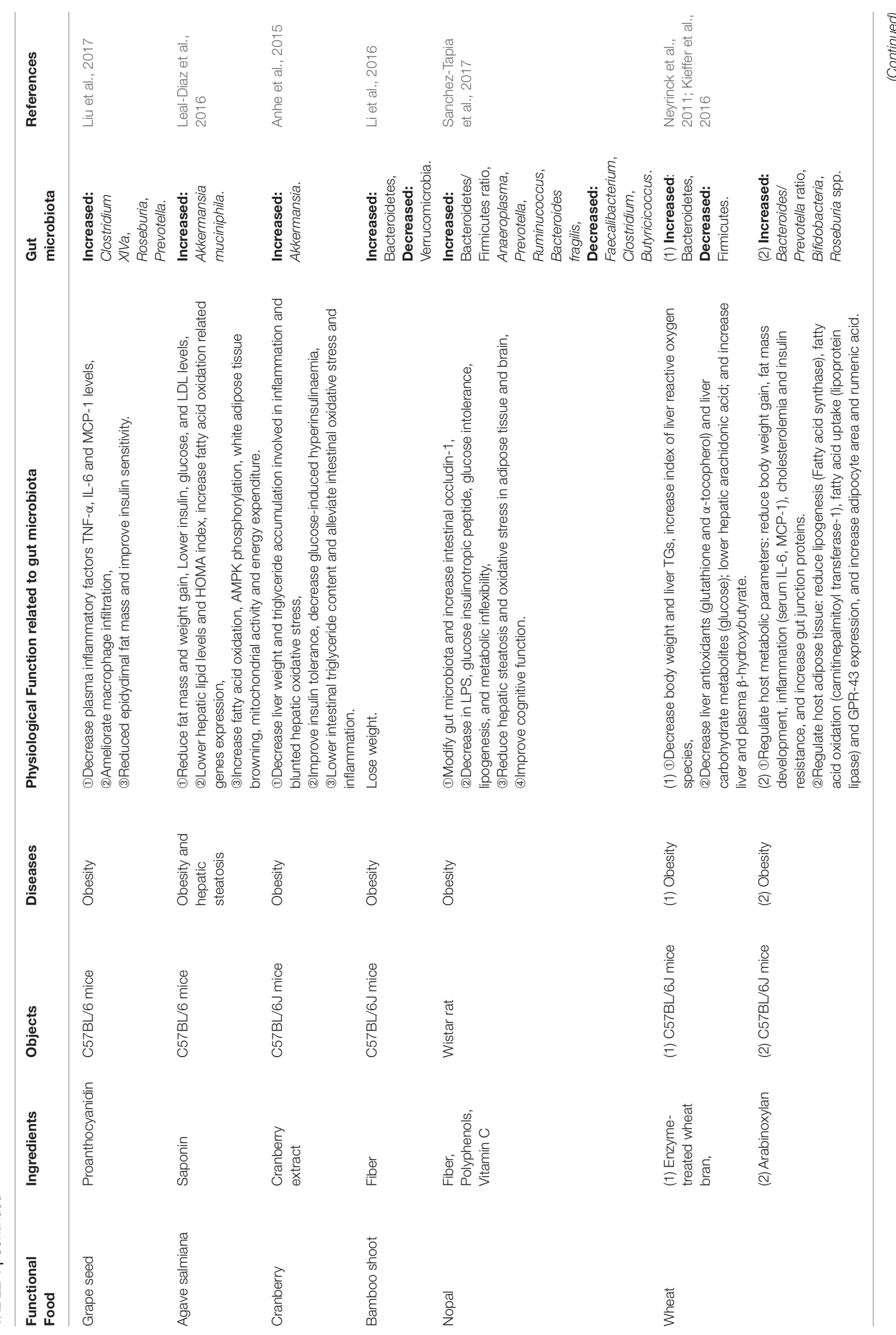




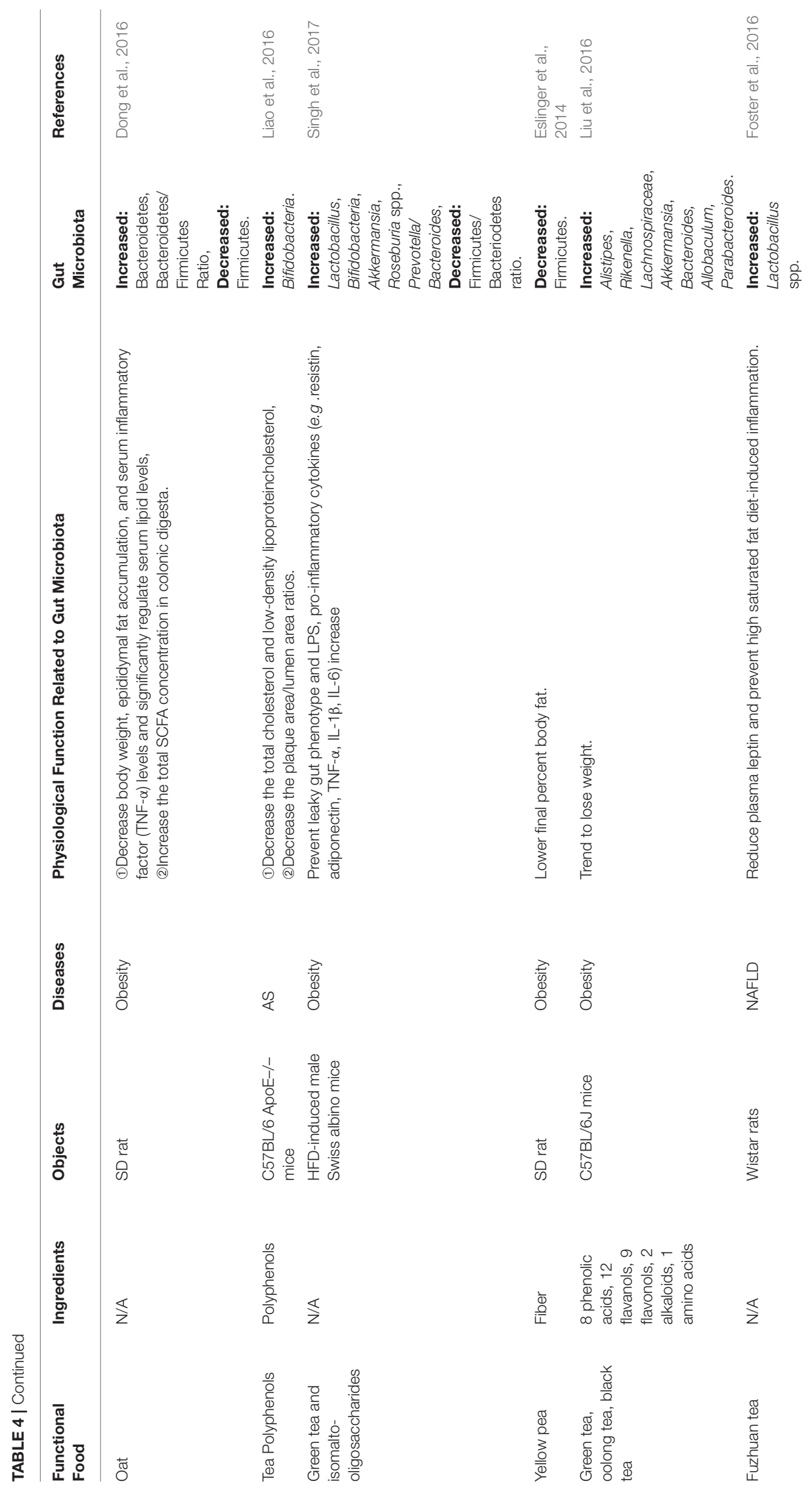


TABLE 5 | Functional food phytochemicals and gut microbiota.

\begin{tabular}{|c|c|c|c|c|c|c|}
\hline Phytochemicals & Category & Objects & Diseases & $\begin{array}{l}\text { Physiological function related to } \\
\text { gut microbiota }\end{array}$ & Gut microbiota & References \\
\hline Laminarin & Polysaccharide & BALB/c mice & Obesity & Reduce energy metabolism & $\begin{array}{l}\text { Increased: } \\
\text { Bacteroidetes } \\
\text { (especially the genus } \\
\text { Bacteroides), } \\
\text { Decreased: } \\
\text { Firmicutes. }\end{array}$ & $\begin{array}{l}\text { Ko et al., 2014; } \\
\text { Nguyen et al., } \\
2016\end{array}$ \\
\hline Fucoidan & Polysaccharide & $\begin{array}{l}\text { C57BL/6J } \\
\text { mice }\end{array}$ & $\begin{array}{l}\text { Intestinal } \\
\text { dysbiosis }\end{array}$ & $\begin{array}{l}\text { Reduce inflammatory response and } \\
\text { antigen load, and decrease } \\
\text { LPS-binding protein levels. }\end{array}$ & $\begin{array}{l}\text { Increased: } \\
\text { Lactobacillus, } \\
\text { Ruminococcaceae, } \\
\text { Decreased: } \\
\text { Peptococcus. }\end{array}$ & Shang et al., 2016 \\
\hline Melatonine & Alkaloid & $\begin{array}{l}\text { C57BL/6J } \\
\text { mice }\end{array}$ & Obesity & Change gut microbiota composition & $\begin{array}{l}\text { Increased: } \\
\text { Akkermansia, } \\
\text { Decreased: } \\
\text { Firmicutes/Bacteroidetes } \\
\text { ratio. }\end{array}$ & Xu P. et al., 2017 \\
\hline Fructans & Polysaccharide & $\begin{array}{l}\text { C57BL/6J } \\
\text { mice }\end{array}$ & Obesity & $\begin{array}{l}\text { Improve intestinal physiology and } \\
\text { shift gut microbiota. }\end{array}$ & $\begin{array}{l}\text { Increased: } \\
\text { Actinobacteria, } \\
\text { Verrucomicrobia } \\
\text { (Akkermansia). }\end{array}$ & $\begin{array}{l}\text { Liu J. P. et al., } \\
2016\end{array}$ \\
\hline $\begin{array}{l}\text { Capsaicin (Chili } \\
\text { peppers) }\end{array}$ & Alkaloid & $\begin{array}{l}\text { C57BL/6J } \\
\text { mice }\end{array}$ & Obesity & $\begin{array}{l}\text { (1)Prevent HFD-induced gut barrier } \\
\text { dysfunction by inhibiting } \\
\text { cannabinoid receptor type } 1 \text { (CB1). } \\
\text { (2)Protect against HFD-induced } \\
\text { obesity is transferrable. }\end{array}$ & $\begin{array}{l}\text { Increased: } \\
\text { Ruminococcaceae, } \\
\text { Lachnospiraceae. }\end{array}$ & Kang et al., 2017 \\
\hline $\begin{array}{l}\text { 3,3-dimethyl-1- } \\
\text { butanol } \\
\text { (DMB) }\end{array}$ & $\begin{array}{l}\text { Choline } \\
\text { analogue }\end{array}$ & $\begin{array}{l}\text { C57BL/6J } \\
\text { mice }\end{array}$ & AS & $\begin{array}{l}\text { Reduce microbial trimethylamine } \\
\text { formation and inhibit choline } \\
\text { diet-enhanced AS. }\end{array}$ & $\mathrm{N} / \mathrm{A}$ & $\begin{array}{l}\text { Wang Q. et al., } \\
2015\end{array}$ \\
\hline
\end{tabular}

Eubacterium, Fecalibacterium and Roseburia are the important members of Firmicutes. Bacteroidetes including the genus Bacteroides, Prevotella and Xylanibacter are known to be efficient degraders of dietary fiber (Simpson and Campbell, 2015). Genus Bifidobacterium is a major member of Actinobacteria. Proteobacteria contains Escherichia and Desulfovibrio, whereas Verrucomicrobia includes only the mucus-degrading genus Akkermansia so far (Schroeder and Backhed, 2016). Tables 1-5 show that the ratio of Firmicutes/Bacteroidetes is modulated in most herbal- and food-intervention studies for CVD as well as various metabolic diseases. For example, decreased ratio of Firmicutes/Bacteroidetes was observed in obesity after intervened by herbals [Daesiho-tang (Hussain et al., 2016), Ganoderma lucidum (Chang C. J. et al., 2015), Flos Lonicera (Wang J. H. et al., 2014), Rhizoma coptidis (Xie et al., 2011), Resveratrol (Qiao et al., 2014), Berberine (Xie et al., 2011; Zhang et al., 2012a), Quercetin (Etxeberria et al., 2015b)] and foods [apple (Jiang et al., 2016; Masumoto et al., 2016), nopal (SanchezTapia et al., 2017), wheat (Kieffer et al., 2016), Laminarin (Nguyen et al., 2016)], as well as in QHD (Feng et al., 2017) and berberine (Cao et al., 2016) for NAFLD (Feng et al., 2017), and barley for CVD (Wang Y. et al., 2016). All of these studies confirmed that increase in gut bacteria phylum Bacteroidetes, and inhibition of Firmicutes, and alteration of Firmicutes/Bacteroidetes ratio helped to treat CMDs including obesity (Ley et al., 2006; Sweeney and Morton, 2013), insulin resistance (Greenhill, 2015),
NAFLD (Liu J. P. et al., 2016) and CVD (Marques et al., 2017). In addition, an increase in the Akkermansia population was found to be in favorable treatment for T2D (Shin et al., 2014), obesity (Everard et al., 2013), AS (Li J. et al., 2016) and some other metabolic syndromes (Roopchand et al., 2015). A recent study showed that fat mass development, insulin resistance and dyslipidemia were reduced by purified membrane protein from Akkermansia (Plovier et al., 2017). Interestingly, the abundance of Akkermansia was dramatically increased not only by Daesiho-tang for T2D (Hussain et al., 2016) and agave salmiana for hepatic steatosis (Leal-Diaz et al., 2016), but also by Flos Lonicera (Wang J. H. et al., 2014), pterostilbene (Etxeberria et al., 2016), apple (Masumoto et al., 2016), grape (Baldwin et al., 2016), agave salmiana (Leal-Diaz et al., 2016), cranberry (Anhe et al., 2015), green tea (Liu Z. et al., 2016; Singh et al., 2017), melatonine (Xu P. et al., 2017) and capsaicin (Kang et al., 2017) for obesity. Moreover, various diseases such as obesity, diabetes and ballergies have been associated with lower numbers of Bifidobacterium at various stages of life (Arboleya et al., 2016). The therapeutical effects of RS for AS (Chen M. L. et al., 2016), berberine for NAFLD (Cao et al., 2016), and Rehmannia glutinosa Libosch (Han et al., 2015), Daesiho-tang (Hussain et al., 2016), rhein (Wang et al., 2016), tea polyphenols (Singh et al., 2017), green tea (Singh et al., 2017) for obesity were associated with the elevated abundance of Bifidobacterium. What's more, increased abundance of genus Lactobacillus, Bacteroides, and 
Prevotella which contribute to metabolic diseases and/or CVD, were also closely associated with digestion of herbals and foods, as shown in Tables 1-5. In summary, increasing the abundance of phylum Bacteroidetes, and genus Akkermansia, Bifidobacterium, Lactobacillus, Bacteroides, and Prevotella, while reducing phylum Firmicutes and Firmicutes/Bacteroidetes ratio may serve as the common characteristics for gut bacteria modulation of herbal medicine and functional food for CMDs. For future studies, the related gut microbiota species interplay with plants and mammalian hosts need to be further investigated.

\section{POTENTIAL EFFECTS OF HERBAL MEDICINE AND FUNCTIONAL FOOD ON GUT-ORGANS AXES}

Commensal gut bacteria impact the host health especially CMDs processes in multiple organs. Several new concepts are proposed in recent reviews focusing on the relationship between gut and organs, such as gut-heart axis (Buglioni and Burnett, 2013), gut-brain axis (De Clercq et al., 2017; Dinan and Cryan, 2017), gut-liver axis (Wiest et al., 2017), gut-kidney axis (Katagiri et al., 2013; Budden et al., 2017) and gut-liverlung axis (Young et al., 2016). The gut is no longer viewed as just a digestive organ, it is also considered as a metabolic and immunomodulatory organ. The major components of fiber, polyphenols and polysaccharides are present in large quantities in both herbal medicine and functianl food, which we have analyzed above. Besides, their muti-ingredient, muti-target and muti-pathway mode are well known and capable of meeting the complex system of the gut-organ interactions. Targetinig the gut-organs axes may also be responsible for CMD treatment. The potential effects are implicated by some latest reports. For instance, a recently published study found that chronic prebiotic treatment indeed exhibited both antidepressant and anxiolytic effects, reduced stress-induced corticosterone release, and modified specific gene expression in the hippocampus and hypothalamus. These effects were exerted via increased cecal acetate and propionate and reduced isobutyrate concentrations. These findings provided clear evidence supporting therapeutic targeting of the gut microbiota for gut-brain axis disorders (Burokas et al., 2017). Another recent finding (Marques et al., 2017) illustrated how HFD and supplementation with acetate influenced gut-heart-kidney axis in a mouse hypertension and heart failure model. It was found that both fiber and acetate decreased gut dysbiosis, measured by the ratio of Firmicutes to Bacteroidetes, and increased the prevalence of Bacteroides acidifaciens. Both HFD and acetate supplementation significantly reduced blood pressure, cardiac fibrosis, and left ventricular hypertrophy. Transcriptome analyses showed that the protective effects of high fiber and acetate were accompanied by the downregulation of cardiac and renal early growth response protein 1 (EGR1), a master cardiovascular regulator involved in cardiac hypertrophy, cardiorenal fibrosis, and inflammation. The upregulation of a network of genes involved in circadian rhythm in both tissues and downregulation of the renin-angiotensin system in the kidney and MAPK signaling in the heart presents an interesting example of gut-multi organs interactions that are simultaneously affected by diet via microbiota.

Althrough there are no reports on herbal medicine or functional food directly targeting gut-organs axes, more study should be carried out in the area to fully exploit the beneficial aspects of gut microbiota. Major components such as fiber and polysaccharide could be fermented and converted into SCFAs which has been shown to be beneficial on the treatment of CMDs. It is foreseeable that the influence of functional food and herbal medicine on the interactive and dynamic relationships between gut microbiota and essential organs will be elucidated in the future.

\section{CONCLUSIONS AND PERSPECTIVE}

The concept of "medicine-food homology" has evolved from its ancient origin and is given a new prospective with newly revealed role of gut microbiota of the host. Human diseases, particularly CMDs, not only could be treated by herbal derived medicine, but also could be prevented by medicinal food via co-inhabiting and influcing gut microbiota.

With the rapid advancement of sequencing technology and intense efforts by researchers, a significant understanding of host gut microbiota has been achieved (Xiao et al., 2017). It is also becoming apparent that herbal medicine and functional food may strongly influence gut microbiota associated with CMDs in humans ranging from obesity to T2D and CVD. Nevertheless, studies on the interaction between herbal derived bioactive compounds and gut microbes are still needed. Future investigation in the field may include, but not limited to the following directions: (1) disease-related and disease-specific microbiota and pathological mechanisms; (2) future research on herbal medicine and functional food should exploit molecular mechanisms and the relationship between microbiota and host behavior; (3) until now, most medicine and functional food research has focused on obesity and T2D rather than CVD, which deserves more careful studies and funding; (4) certain polyphenols (puerarin, paeoniflorin, baicalein, icariin, mangiferin, gallic acid, luteolin, cryptotanshinone, kaempferol, etc.) are similar to RSV and showed poor absorption into the bloodstream after oral administration, but they may have an impact on gut microbiota as well. In conclusion (Figure 1), herbal medicine and functional food with major ingredients including fiber, polyphenols and polysaccharides are inclined to increasing abundance of phylum Bacteroidetes, and genus Akkermansia, Bifidobacterium, Lactobacillus, Bacteroides, and Prevotella, while reducing phylum Firmicutes and Firmicutes/Bacteroidetes ratio to prevent or treat CMDs through SCFAs, BAs, LPS and TMAO signaling. The condition of health or disease in human is critically dependent on the balance between medicine/food by modulating gut microbiota. Human intake of herbal medicine and functional food can alter gut microbiota, and microbiome in turn can influce human health through microbial metabolites. The convergence between herbal medicine and functional food through microbiota reinforces the idea that CMDs are not 


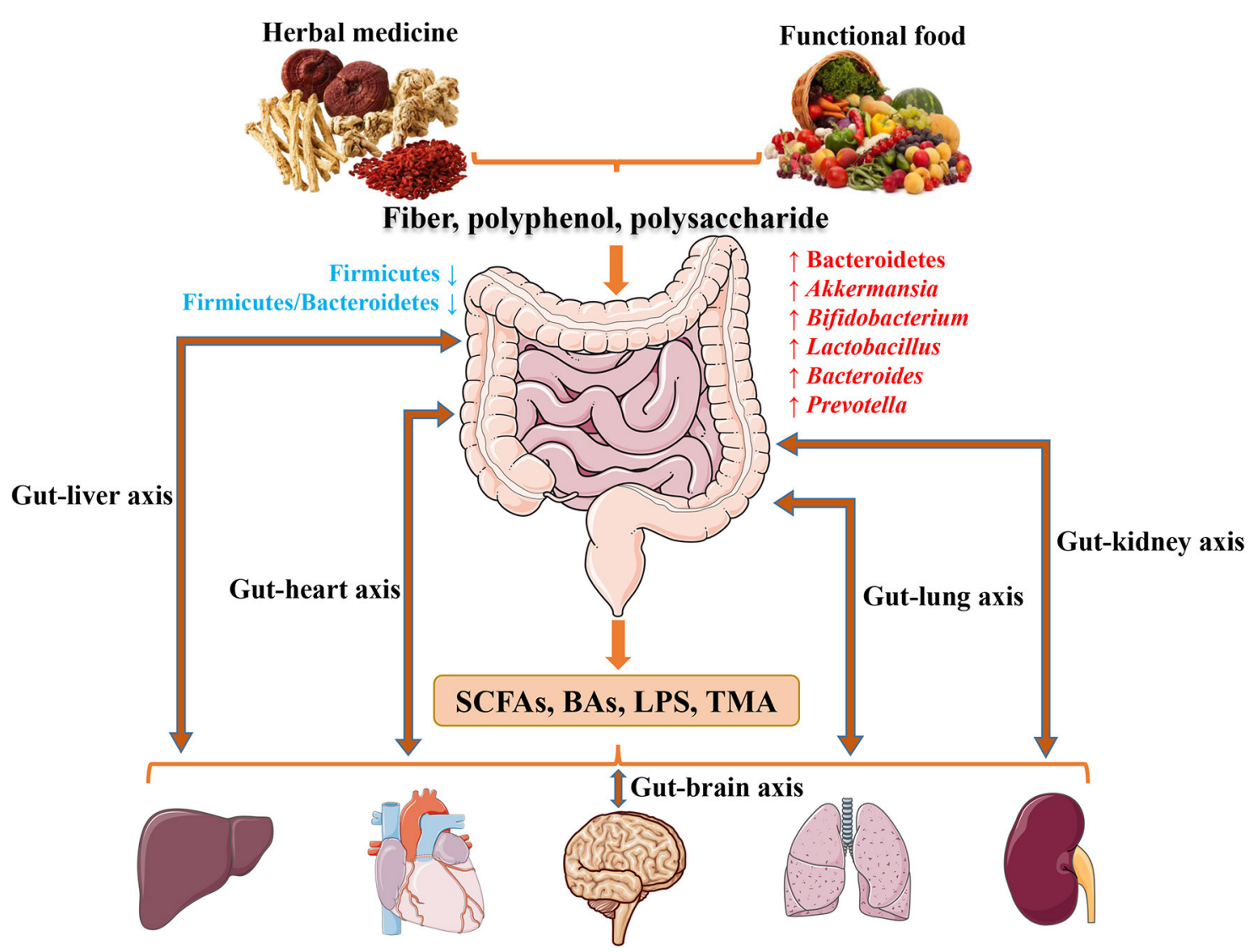

FIGURE 1 | The potentially shared biological processes and underlying mechanisms of herbal medicine and functional food for CMDs through modulating microbiota.

only treatable but also preventable by maintaining the balance between the two.

\section{AUTHOR CONTRIBUTIONS}

YZ conceived, designed, organizedand revised the manuscript; ML conceived, designed, wrote and revised the manuscript; YW, GF, XW, and SX revised the manuscript and discussed interpretation.

\section{REFERENCES}

Anhe, F. F., Roy, D., Pilon, G., Dudonne, S., Matamoros, S., Varin, T. V., et al. (2015). A polyphenol-rich cranberry extract protects from diet-induced obesity, insulin resistance and intestinal inflammation in association with increased Akkermansia spp. population in the gut microbiota of mice. Gut 64, 872-883. doi: 10.1136/gutjnl-2014-307142

Arboleya, S., Watkins, C., Stanton, C., and Ross, R. P. (2016). Gut Bifidobacteria Populations in Human Health and Aging. Front. Microbiol. 7:1204. doi: $10.3389 /$ fmicb.2016.01204

Aron-Wisnewsky, J., and Clement, K. (2016). The gut microbiome, diet, and links to cardiometabolic and chronic disorders. Nat. Rev. Nephrol. 12, 169-181. doi: 10.1038/nrneph.2015.191

Baldwin, J., Collins, B., Wolf, P. G., Martinez, K., Shen, W., Chuang, C. C., et al. (2016). Table grape consumption reduces adiposity and markers of hepatic

\section{ACKNOWLEDGMENTS}

This study was supported by grants from National Science and Technology Major Projects for "Major New Drugs Innovation and Development" (Nos. 2013ZX09201020 and 2015ZX09J15102-004-004), the National Science Foundation of China (NSFC 81274128), and the International Cooperation Project of MOST, China (2013DFA31620). We thank John Orgah for his expert editorial assistance.

lipogenesis and alters gut microbiota in butter fat-fed mice. J. Nutr. Biochem. 27, 123-135. doi: 10.1016/j.jnutbio.2015.08.027

Baur, J. A., and Sinclair, D. A. (2006). Therapeutic potential of resveratrol: the in vivo evidence. Nat. Rev. Drug Discov. 5, 493-506. doi: 10.1038/ $\operatorname{nrd} 2060$

Bennett, B. J., De Aguiar Vallim, T. Q., Wang, Z., Shih, D. M., Meng, Y., Gregory, J., et al. (2013). Trimethylamine-N-oxide, a metabolite associated with atherosclerosis, exhibits complex genetic and dietary regulation. Cell Metab. 17, 49-60. doi: 10.1016/j.cmet.2012.12.011

Bindels, L. B., Delzenne, N. M., Cani, P. D., and Walter, J. (2015). Towards a more comprehensive concept for prebiotics. Nat. Rev. Gastroenterol. Hepatol. 12, 303-310. doi: 10.1038/nrgastro.2015.47

Bordin, M., D'atri, F., Guillemot, L., and Citi, S. (2004). Histone deacetylase inhibitors up-regulate the expression of tight junction proteins. Mol. Cancer Res. 2, 692-701. 
Budden, K. F., Gellatly, S. L., Wood, D. L., Cooper, M. A., Morrison, M., Hugenholtz, P., et al. (2017). Emerging pathogenic links between microbiota and the gut-lung axis. Nat. Rev. Microbiol. 15, 55-63. doi: $10.1038 /$ nrmicro.2016.142

Buglioni, A., and Burnett, J. C. Jr. (2013). A gut-heart connection in cardiometabolic regulation. Nat. Med. 19, 534-536. doi: 10.1038/nm.3196

Burokas, A., Arboleya, S., Moloney, R. D., Peterson, V. L., Murphy, K., Clarke, G., et al. (2017). Targeting the Microbiota-Gut-Brain Axis: prebiotics have anxiolytic and antidepressant-like effects and reverse the impact of chronic stress in mice. Biol Psychiatry. 82, 472-487. doi: 10.1016/j.biopsych.2016.12.031

Canfora, E. E., Jocken, J. W., and Blaak, E. E. (2015). Short-chain fatty acids in control of body weight and insulin sensitivity. Nat. Rev. Endocrinol. 11, 577-591. doi: 10.1038/nrendo.2015.128

Cani, P. D., Amar, J., Iglesias, M. A., Poggi, M., Knauf, C., Bastelica, D., et al. (2007). Metabolic endotoxemia initiates obesity and insulin resistance. Diabetes 56, 1761-1772. doi: 10.2337/db06-1491

Cani, P. D., Bibiloni, R., Knauf, C., Waget, A., Neyrinck, A. M., Delzenne, N. M., et al. (2008). Changes in gut microbiota control metabolic endotoxemiainduced inflammation in high-fat diet-induced obesity and diabetes in mice. Diabetes 57, 1470-1481. doi: 10.2337/db07-1403

Cao, Y., Pan, Q., Cai, W., Shen, F., Chen, G. Y., Xu, L. M., et al. (2016). Modulation of gut microbiota by berberine improves steatohepatitis in high-fat diet-fed BALB/C mice. Arch. Iran. Med. 19, 197-203.

Carroll, S. P., Jorgensen, P. S., Kinnison, M. T., Bergstrom, C. T., Denison, R. F., Gluckman, P., et al. (2014). Applying evolutionary biology to address global challenges. Science 346:1245993. doi: 10.1126/science.1245993

Chang, C. J., Lin, C. S., Lu, C. C., Martel, J., Ko, Y. F., Ojcius, D. M., et al. (2015). Ganoderma lucidum reduces obesity in mice by modulating the composition of the gut microbiota. Nat. Commun. 6:7489. doi: 10.1038/ ncomms 8489

Chen, M.-L., Yi, L., Zhang, Y., Zhou, X., Ran, L., Yang, J., et al. (2016). Resveratrol attenuates trimethylamine- $N$-Oxide (TMAO)-induced atherosclerosis by regulating TMAO synthesis and bile acid metabolism via remodeling of the gut microbiota. MBio 7:e02210-15. doi: 10.1128/mBio.02210-15

Chen, X., Lou, G., Meng, Z., and Huang, W. (2011). TGR5: a novel target for weight maintenance and glucose metabolism. Exp. Diab. Res. 2011:853501. doi: 10.1155/2011/853501

Chen, Y. M., Liu, Y., Zhou, R. F., Chen, X. L., Wang, C., Tan, X. Y., et al. (2016). Associations of gut-flora-dependent metabolite trimethylamine-Noxide, betaine and choline with non-alcoholic fatty liver disease in adults. Sci. Rep. 6:19076. doi: 10.1038/srep19076

Cho, I., Yamanishi, S., Cox, L., Methe, B. A., Zavadil, J., Li, K., et al. (2012). Antibiotics in early life alter the murine colonic microbiome and adiposity. Nature 488, 621-626. doi: 10.1038/nature11400

Claus, S. P. (2017). Will gut microbiota help design the next generation of GLP-1-based therapies for Type 2 diabetes? Cell Metab. 26, 6-7. doi: 10.1016/j.cmet.2017.06.009

Craciun, S., and Balskus, E. P. (2012). Microbial conversion of choline to trimethylamine requires a glycyl radical enzyme. Proc. Natl. Acad. Sci. U.S.A. 109, 21307-21312. doi: 10.1073/pnas.1215689109

Craciun, S., Marks, J. A., and Balskus, E. P. (2014). Characterization of choline trimethylamine-lyase expands the chemistry of glycyl radical enzymes. ACS Chem. Biol. 9, 1408-1413. doi: 10.1021/cb500113p

Creely, S. J., Mcternan, P. G., Kusminski, C. M., Fisher, F., M., Da Silva, N. F., Khanolkar, M., et al. (2007). Lipopolysaccharide activates an innate immune system response in human adipose tissue in obesity and type 2 diabetes. Am. J. Physiol. Endocrinol. Metab. 292, E740-E747. doi: 10.1152/ajpendo.0 0302.2006

Cuervo, A., Hevia, A., Lopez, P., Suarez, A., Sanchez, B., Margolles, A., et al. (2015). Association of polyphenols from oranges and apples with specific intestinal microorganisms in systemic lupus erythematosus patients. Nutrients 7, 1301-1317. doi: 10.3390/nu7021301

Dambrova, M., Latkovskis, G., Kuka, J., Strele, I., Konrade, I., Grinberga, S., et al. (2016). Diabetes is Associated with Higher Trimethylamine N-oxide Plasma Levels. Exp. Clin. Endocrinol. Diab. 124, 251-256. doi: 10.1055/s-0035-1569330

De Clercq, N., Frissen, M. N., Groen, A. K., and Nieuwdorp, M. (2017). Gut Microbiota and the Gut-Brain Axis: new insights in the pathophysiology of metabolic syndrome. Psychosom Med. 79, 874-879. doi: 10.1097/PSY.0000000000000495
Dinan, T. G., and Cryan, J. F. (2017). Gut-brain axis in 2016: brain-gut-microbiota axis - mood, metabolism and behaviour. Nat. Rev. Gastroenterol. Hepatol. 14, 69-70. doi: 10.1038/nrgastro.2016.200

Dong, J. L., Zhu, Y. Y., Ma, Y. L., Xiang, Q. S., Shen, R. L., and Liu, Y. Q. (2016). Oat products modulate the gut microbiota and produce anti-obesity effects in obese rats. J. Funct. Foods 25, 408-420. doi: 10.1016/j.jff.2016.06.025

Eslinger, A. J., Eller, L. K., and Reimer, R. A. (2014). Yellow pea fiber improves glycemia and reduces Clostridium leptum in diet-induced obese rats. Nutr. Res. 34, 714-722. doi: 10.1016/j.nutres.2014.07.016

Etxeberria, U., Arias, N., Boque, N., Macarulla, M. T., Portillo, M. P., Martinez, J. A., et al. (2015a). Reshaping faecal gut microbiota composition by the intake of trans-resveratrol and quercetin in high-fat sucrose diet-fed rats. J. Nutr. Biochem. 26, 651-660. doi: 10.1016/j.jnutbio.2015.01.002

Etxeberria, U., Arias, N., Boque, N., Romo-Hualde, A., Macarulla, M. T., Portillo, M. P., et al. (2015b). Metabolic faecal fingerprinting of transresveratrol and quercetin following a high-fat sucrose dietary model using liquid chromatography coupled to high-resolution mass spectrometry. Food Funct. 6, 2758-2767. doi: 10.1039/C5FO00473J

Etxeberria, U., Hijona, E., Aguirre, L., Milagro, F. I., Bujanda, L., Rimando, A. M., et al. (2016). Pterostilbene-induced changes in gut microbiota composition in relation to obesity. Mol. Nutr. Food Res. 61:1500906. doi: $10.1002 / \mathrm{mnfr} .201500906$

Everard, A., Belzer, C., Geurts, L., Ouwerkerk, J. P., Druart, C., Bindels, L. B., et al. (2013). Cross-talk between Akkermansia muciniphila and intestinal epithelium controls diet-induced obesity. Proc. Natl. Acad. Sci. U.S.A. 110, 9066-9071. doi: 10.1073/pnas.1219451110

Fang, W., Wei, C., Dong, Y., Tang, X., and Zu, Y. (2016). The effect on gut microbiota structure of primarily diagnosed type 2 diabetes patients intervened by sancai lianmei particle and acarbose: a randomized controlled trial. J. Clin. Trails 6:270. doi: 10.4172/2167-0870.1000270

Feng, Q., Liu, W., Baker, S. S., Li, H., Chen, C., Liu, Q., et al. (2017). Multitargeting therapeutic mechanisms of the Chinese herbal medicine QHD in the treatment of non-alcoholic fatty liver disease. Oncotarget 8, 27820-27838. doi: 10.18632 /oncotarget.15482

Foster, M. T., Gentile, C. L., Cox-York, K., Wei, Y., Wang, D., Estrada, A. L., et al. (2016). Fuzhuan tea consumption imparts hepatoprotective effects and alters intestinal microbiota in high saturated fat diet-fed rats. Mol. Nutr. Food Res. 60, 1213-1220. doi: 10.1002/mnfr.201500654

Ghosh, S. S., Bie, J. H., Wang, J., and Ghosh, S. (2014). Oral Supplementation with Non-Absorbable Antibiotics or Curcumin Attenuates Western DietInduced Atherosclerosis and Glucose Intolerance in LDLR-/- Mice - Role of Intestinal Permeability and Macrophage Activation. PLoS ONE 9:0108577. doi: 10.1371/journal.pone.0108577

Grasset, E., Puel, A., Charpentier, J., Collet, X., Christensen, J. E., Terce, F., et al. (2017). A Specific Gut Microbiota Dysbiosis of Type 2 Diabetic Mice Induces GLP-1 Resistance through an Enteric NO-Dependent and Gut-Brain Axis Mechanism. Cell Metab. 26:278. doi: 10.1016/j.cmet.2017.04.013

Greenhill, C. (2015). Gut microbiota: firmicutes and bacteroidetes involved in insulin resistance by mediating levels of glucagon-like peptide 1. Nat. Rev. Endocrinol. 11:254. doi: 10.1038/nrendo.2015.40

Greenhill, C. (2016). Metabolism: intestinal microbiota affects host physiology. Nat. Rev. Endocrinol. 13:64. doi: 10.1038/nrendo.2016.207

Guarner, F., and Malagelada, J. R. (2003). Gut flora in health and disease. Lancet 361, 512-519. doi: 10.1016/S0140-6736(03)12489-0

Guo, M., Ding, S., Zhao, C., Gu, X., He, X., Huang, K., et al. (2015). Red Ginseng and Semen Coicis can improve the structure of gut microbiota and relieve the symptoms of ulcerative colitis. J. Ethnopharmacol. 162, 7-13. doi: 10.1016/j.jep.2014.12.029

Han, K., Bose, S., Kim, Y. M., Chin, Y. W., Kim, B. S., Wang, J. H., et al. (2015) Rehmannia glutinosa reduced waist circumferences of Korean obese women possibly through modulation of gut microbiota. Food Funct. 6, 2684-2692. doi: 10.1039/C5FO00232J

Hansen, T. H., Gobel, R. J., Hansen, T., and Pedersen, O. (2015). The gut microbiome in cardio-metabolic health. Genome Med 7:33. doi: 10.1186/s13073-015-0157-z

Heianza, Y., Ma, W., Manson, J. E., Rexrode, K. M., and Qi, L. (2017). Gut microbiota metabolites and risk of major adverse cardiovascular disease events and death: a systematic review and meta-analysis of prospective studies. J. Am. Heart Assoc. 6:e004947. doi: 10.1161/JAHA.116.004947 
Hennessy, E. J., Parker, A. E., and O'neill, L. A. (2010). Targeting Tolllike receptors: emerging therapeutics? Nat. Rev. Drug Discov. 9, 293-307. doi: $10.1038 / \operatorname{nrd} 3203$

Henning, S. M., Yang, J., Shao, P., Lee, R. P., Huang, J., Ly, A., et al. (2017). Health benefit of vegetable/fruit juice-based diet: role of microbiome. Sci. Rep. 7:2167. doi: 10.1038/s41598-017-02200-6

Heo, J., Seo, M., Park, H., Lee, W. K., Guan, L. L., Yoon, J., et al. (2016). Gut microbiota modulated by probiotics and garcinia cambogia extract correlate with weight gain and adipocyte sizes in high fat-fed mice. Sci. Rep. 6:33566. doi: $10.1038 /$ srep 33566

Heyman-Linden, L., Kotowska, D., Sand, E., Bjursell, M., Plaza, M., Turner, C., et al. (2016). Lingonberries alter the gut microbiota and prevent lowgrade inflammation in high-fat diet fed mice. Food Nutr. Res. 60:29993. doi: 10.3402/fnr.v60.29993

Hu, Y., Zhao, Y., Yuan, L., and Yang, X. (2015). Protective effects of tartary buckwheat flavonoids on high TMAO diet-induced vascular dysfunction and liver injury in mice. Food Funct 6, 3359-3372. doi: 10.1039/C5FO00581G

Human Microbiome Project, C. (2012). A framework for human microbiome research. Nature 486, 215-221. doi: 10.1038/nature11209

Hussain, A., Yadav, M. K., Bose, S., Wang, J. H., Lim, D., Song, Y. K., et al. (2016). Daesiho-Tang is an effective herbal formulation in attenuation of obesity in mice through alteration of gene expression and modulation of intestinal microbiota. PLoS ONE 11:e0165483. doi: 10.1371/journal.pone.0165483

Inagaki, T., Choi, M., Moschetta, A., Peng, L., Cummins, C. L., Mcdonald, J. G., et al. (2005). Fibroblast growth factor 15 functions as an enterohepatic signal to regulate bile acid homeostasis. Cell Metab. 2, 217-225. doi: 10.1016/j.cmet.2005.09.001

Ishizaki, K., Imada, T., and Tsurufuji, M. (2005). Hepatoprotective bile acid 'ursodeoxycholic acid (UDCA)' Property and difference as bile acids. Hepatol. Res. 33, 174-177. doi: 10.1016/j.hepres.2005.09.029

Janssen, A. W., and Kersten, S. (2015). The role of the gut microbiota in metabolic health. FASEB J. 29, 3111-3123. doi: 10.1096/fj.14-269514

Jiang, T., Gao, X., Wu, C., Tian, F., Lei, Q., Bi, J., et al. (2016). Apple-derived pectin modulates gut microbiota, improves gut barrier function, and attenuates metabolic endotoxemia in rats with diet-induced obesity. Nutrients 8:126. doi: $10.3390 /$ nu 8030126

Jorgensen, P. S., Wernli, D., Carroll, S. P., Dunn, R. R., Harbarth, S., Levin, S. A., et al. (2016). Use antimicrobials wisely. Nature 537, 159-161. doi: $10.1038 / 537159$ a

Kafantaris, I., Kotsampasi, B., Christodoulou, V., Kokka, E., Kouka, P., Terzopoulou, Z., et al. (2016). Grape pomace improves antioxidant capacity and faecal microflora of lambs. J. Anim. Physiol. Anim. Nutr. (Berl). 101, e108-e121. doi: 10.1111/jpn. 12569

Kang, C., Wang, B., Kaliannan, K., Wang, X., Lang, H., Hui, S., et al. (2017). Gut microbiota mediates the protective effects of dietary capsaicin against chronic low-grade inflammation and associated obesity induced by high-fat diet. MBio 8:e0470-17. doi: 10.1128/mBio.00470-17

Kang, Y. E., Kim, J. M., Joung, K. H., Lee, J. H., You, B. R., Choi, M. J., et al. (2016). The Roles of Adipokines, Proinflammatory Cytokines, and Adipose Tissue Macrophages in Obesity-Associated Insulin Resistance in Modest Obesity and Early Metabolic Dysfunction. PLOS ONE 11:e0154003. doi: 10.1371/journal.pone. 0154003

Katagiri, D., Hamasaki, Y., Doi, K., Okamoto, K., Negishi, K., Nangaku, M., et al. (2013). Protection of glucagon-like peptide-1 in cisplatin-induced renal injury elucidates gut-kidney connection. J. Am. Soc. Nephrol. 24, 2034-2043. doi: 10.1681/ASN.2013020134

Kieffer, D. A., Piccolo, B. D., Marco, M. L., Kim, E. B., Goodson, M. L., Keenan, M. J., et al. (2016). Obese mice fed a diet supplemented with enzyme-treated wheat bran display marked shifts in the liver metabolome concurrent with altered gut bacteria. J. Nutr. 146, 2445-2460. doi: 10.3945/jn.116.238923

Kimura, I., Ozawa, K., Inoue, D., Imamura, T., Kimura, K., Maeda, T., et al. (2013). The gut microbiota suppresses insulin-mediated fat accumulation via the short-chain fatty acid receptor GPR43. Nat. Commun. 4:1829. doi: $10.1038 /$ ncomms 2852

Kitai, T., and Tang, W. H. W. (2017). The role and impact of gut microbiota in cardiovascular disease. Rev. Esp. Cardiol. (Engl. Ed). 70, 799-800. doi: 10.1016/j.recesp.2017.04.003
Klinder, A., Shen, Q., Heppel, S., Lovegrove, J. A., Rowland, I., and Tuohy, K. M. (2016). Impact of increasing fruit and vegetables and flavonoid intake on the human gut microbiota. Food Funct. 7, 1788-1796. doi: 10.1039/C5FO01096A

Ko, S. J., Kim, J., Han, G., Kim, S. K., Kim, H. G., Yeo, I., et al. (2014). Laminaria japonica combined with probiotics improves intestinal microbiota: a randomized clinical trial. J. Med. Food 17, 76-82. doi: 10.1089/jmf.2013.3054

Koeth, R. A., Wang, Z., Levison, B. S., Buffa, J. A., Org, E., Sheehy, B. T., et al (2013). Intestinal microbiota metabolism of L-carnitine, a nutrient in red meat, promotes atherosclerosis. Nat. Med. 19, 576-585. doi: 10.1038/nm.3145

Koopen, A. M., Groen, A. K., and Nieuwdorp, M. (2016). Human microbiome as therapeutic intervention target to reduce cardiovascular disease risk. Curr. Opin. Lipidol. 27, 615-622. doi: 10.1097/MOL.0000000000000357

Koutsos, A., Tuohy, K. M., and Lovegrove, J. A. (2015). Apples and cardiovascular health-is the gut microbiota a core consideration? Nutrients 7, 3959-3998. doi: 10.3390/nu7063959

Laparra, J. M., and Sanz, Y. (2010). Interactions of gut microbiota with functional food components and nutraceuticals. Pharmacol. Res. 61, 219-225. doi: 10.1016/j.phrs.2009.11.001

Laxminarayan, R., Matsoso, P., Pant, S., Brower, C., Rottingen, J. A., Klugman, K., et al. (2016). Access to effective antimicrobials: a worldwide challenge. Lancet 387, 168-175. doi: 10.1016/S0140-6736(15)00474-2

Leal-Diaz, A. M., Noriega, L. G., Torre-Villalvazo, I., Torres, N., AlemanEscondrillas, G., Lopez-Romero, P., et al. (2016). Aguamiel concentrate from Agave salmiana and its extracted saponins attenuated obesity and hepatic steatosis and increased Akkermansia muciniphila in C57BL6 mice. Sci. Rep. 6:34242. doi: $10.1038 /$ srep34242

Ley, R. E., Turnbaugh, P. J., Klein, S., and Gordon, J. I. (2006). Microbial ecology: human gut microbes associated with obesity. Nature 444, 1022-1023. doi: $10.1038 / 4441022$ a

Li, F., Jiang, C., Krausz, K. W., Li, Y., Albert, I., Hao, H., et al. (2013). Microbiome remodelling leads to inhibition of intestinal farnesoid $\mathrm{X}$ receptor signalling and decreased obesity. Nat. Commun. 4:2384. doi: 10.1038/ncomms3384

Li, J., Lin, S., Vanhoutte, P. M., Woo, C. W., and Xu, A. (2016). Akkermansia Muciniphila Protects Against Atherosclerosis by Preventing Metabolic Endotoxemia-Induced Inflammation in Apoe ${ }^{-/-}$Mice. Circulation 133, 2434-2446. doi: 10.1161/CIRCULATIONAHA.115.019645

Li, T., Chen, Y., Gua, C., and Li, X. (2017). Elevated circulating trimethylamine $\mathrm{N}$-Oxide levels contribute to endothelial dysfunction in aged rats through vascular inflammation and oxidative stress. Front. Physiol. 8:350. doi: 10.3389/fphys.2017.00350

Li, X. S., Obeid, S., Klingenberg, R., Gencer, B., Mach, F., Raber, L., et al. (2017). Gut microbiota-dependent trimethylamine $\mathrm{N}$-oxide in acute coronary syndromes: a prognostic marker for incident cardiovascular events beyond traditional risk factors. Eur. Heart J. 38, 814-824. doi: 10.1093/eurheartj/ehw582

Li, X., Guo, J., Ji, K., and Zhang, P. (2016). Bamboo shoot fiber prevents obesity in mice by modulating the gut microbiota. Sci. Rep. 6:32953. doi: 10.1038/srep32953

Li, Y., Yao, J., Han, C., Yang, J., Chaudhry, M. T., Wang, S., et al. (2016). Quercetin, inflammation and immunity. Nutrients 8:167. doi: 10.3390/nu80 30167

Liao, Z. L., Zeng, B. H., Wang, W., Li, G. H., Wu, F., Wang, L., et al. (2016). Impact of the Consumption of Tea Polyphenols on Early Atherosclerotic Lesion Formation and Intestinal Bifidobacteria in High-Fat-Fed $\mathrm{ApoE}^{-/-}$Mice. Front. Nutr. 3:42. doi: 10.3389/fnut.2016.00042

Liu, J. P., Zou, W. L., Chen, S. J., Wei, H. Y., Yin, Y. N., Zou, Y. Y., et al. (2016). Effects of different diets on intestinal microbiota and nonalcoholic fatty liver disease development. World J. Gastroenterol. 22, 7353-7364. doi: 10.3748/wjg.v22.i32.7353

Liu, T. W., Cephas, K. D., Holscher, H. D., Kerr, K. R., Mangian, H. F., Tappenden, K. A., et al. (2016). Nondigestible fructans alter gastrointestinal barrier function, gene expression, histomorphology, and the microbiota profiles of diet-induced obese C57BL/6J Mice. J. Nutr. 146, 949-956. doi: $10.3945 /$ jn.115.227504

Liu, W., Zhao, S., Wang, J., Shi, J., Sun, Y., Wang, W., et al. (2017). Grape seed proanthocyanidin extract ameliorates inflammation and adiposity by modulating gut microbiota in high-fat diet mice. Mol. Nutr. Food Res. 61:1601082. doi: 10.1002/mnfr.201601082 
Liu, Z., Chen, Z., Guo, H., He, D., Zhao, H., Wang, Z., et al. (2016). The modulatory effect of infusions of green tea, oolong tea, and black tea on gut microbiota in high-fat-induced obese mice. Food Funct. 7, 4869-4879. doi: 10.1039/C6FO01439A

Ma, G., Pan, B., Chen, Y., Guo, C., Zhao, M., Zheng, L., et al. (2017). Trimethylamine $\mathrm{N}$-oxide in atherogenesis: impairing endothelial Self-repair capacity and enhancing monocyte adhesion. Biosci Rep. 37:BSR20160244. doi: 10.1042/BSR20160244

Makishima, M., Okamoto, A. Y., Repa, J. J., Tu, H., Learned, R. M., Luk, A., et al. (1999). Identification of a nuclear receptor for bile acids. Science 284, 1362-1365. doi: 10.1126/science.284.5418.1362

Marques, F. Z., Nelson, E., Chu, P. Y., Horlock, D., Fiedler, A., Ziemann, M., et al. (2017). High-fiber diet and acetate supplementation change the gut microbiota and prevent the development of hypertension and heart failure in hypertensive mice. Circulation 135, 964-977. doi: 10.1161/CIRCULATIONAHA.116.024545

Martel, J., Ojcius, D. M., Chang, C. J., Lin, C. S., Lu, C. C., Ko, Y. F., et al. (2016). Anti-obesogenic and antidiabetic effects of plants and mushrooms. Nat. Rev. Endocrinol. 13, 149-160. doi: 10.1038/nrendo.2016.142

Maruyama, T., Miyamoto, Y., Nakamura, T., Tamai, Y., Okada, H., Sugiyama, E., et al. (2002). Identification of membrane-type receptor for bile acids (M-BAR). Biochem. Biophys. Res. Commun. 298, 714-719. doi: 10.1016/S0006-291X(02)02550-0

Masumoto, S., Terao, A., Yamamoto, Y., Mukai, T., Miura, T., and Shoji, T. (2016). Non-absorbable apple procyanidins prevent obesity associated with gut microbial and metabolomic changes. Sci. Rep. 6:31208. doi: 10.1038/ srep31208

Mayerhofer, C. C. K., Ueland, T., Broch, K., Vincent, R. P., Cross, G. F., Dahl, C. P., et al. (2017). Increased Secondary/Primary Bile Acid-Ratio in Chronic Heart Failure. J Card Fail. 23, 666-671. doi: 10.1016/j.cardfail.2017.06.007

Mcfadden, R. M., Larmonier, C. B., Shehab, K. W., Midura-Kiela, M., Ramalingam, R., Harrison, C. A., et al. (2015). The role of curcumin in modulating colonic microbiota during colitis and colon cancer prevention. Inflamm. Bowel Dis. 21, 2483-2494. doi: 10.1097/MIB.0000000000000522

Mei, L., Tang, Y., Li, M., Yang, P., Liu, Z., Yuan, J., et al. (2015). CoAdministration of Cholesterol-Lowering Probiotics and Anthraquinone from Cassia obtusifolia L. Ameliorate Non-Alcoholic Fatty Liver. PLoS ONE 10:e0138078. doi: 10.1371/journal.pone.0138078

Meyer, K. A., and Bennett, B. J. (2016). Diet and gut microbial function in metabolic and cardiovascular disease risk. Curr. Diab. Rep. 16:93. doi: $10.1007 / \mathrm{s} 11892-016-0791-\mathrm{x}$

Micha, R., Penalvo, J. L., Cudhea, F., Imamura, F., Rehm, C. D., and Mozaffarian, D. (2017). Association Between Dietary Factors and Mortality From Heart Disease, Stroke, and Type 2 Diabetes in the United States. JAMA 317, 912-924. doi: 10.1001/jama.2017.0947

Midtvedt, T. (1974). Microbial bile acid transformation. Am. J. Clin. Nutr. 27, 1341-1347.

Moreno-Indias, I., Cardona, F., Tinahones, F. J., and Queipo-Ortuno, M. I. (2014). Impact of the gut microbiota on the development of obesity and type 2 diabetes mellitus. Front. Microbiol. 5:190. doi: 10.3389/fmicb.2014.00190

Most, J., Penders, J., Lucchesi, M., Goossens, G. H., and Blaak, E. E. (2017). Gut microbiota composition in relation to the metabolic response to 12 -week combined polyphenol supplementation in overweight men and women. Eur. J. Clin. Nutr. 71, 1040-1045. doi: 10.1038/ejcn.2017.89

Muccioli, G. G., Naslain, D., Backhed, F., Reigstad, C. S., Lambert, D. M., Delzenne, N. M., et al. (2010). The endocannabinoid system links gut microbiota to adipogenesis. Mol. Syst. Biol. 6:392. doi: 10.1038/msb.2010.46

Mueller, M., Thorell, A., Claudel, T., Jha, P., Koefeler, H., Lackner, C., et al. (2015). Ursodeoxycholic acid exerts farnesoid X receptor-antagonistic effects on bile acid and lipid metabolism in morbid obesity. J. Hepatol. 62, 1398-1404. doi: 10.1016/j.jhep.2014.12.034

Neyrinck, A. M., Possemiers, S., Druart, C., Van De Wiele, T., De Backer, F., Cani, P. D., et al. (2011). Prebiotic effects of wheat arabinoxylan related to the increase in bifidobacteria, Roseburia and Bacteroides/Prevotella in diet-induced obese mice. PLoS ONE 6:e20944. doi: 10.1371/journal.pone.0020944

Nguyen, S. G., Kim, J., Guevarra, R. B., Lee, J.-H., Kim, E., Kim, S.-I., et al. (2016). Laminarin favorably modulates gut microbiota in mice fed a high-fat diet. Food Funct. 7, 4193-4201. doi: 10.1039/C6FO00929H
Oellgaard, J., Winther, S. A., Hansen, T. S., Rossing, P., and Von Scholten, B. J. (2017). Trimethylamine N-oxide (TMAO) as a new potential therapeutic target for insulin resistance and cancer. Curr Pharm Des. 23, 3699-3712. doi: $10.2174 / 1381612823666170622095324$

Organ, C. L., Otsuka, H., Bhushan, S., Wang, Z., Bradley, J., Trivedi, R., et al. (2016). Choline Diet and Its Gut Microbe-Derived Metabolite, Trimethylamine N-Oxide, Exacerbate Pressure Overload-Induced Heart Failure. Circ. Heart Fail 9:e002314. doi: 10.1161/CIRCHEARTFAILURE.115.002314

Parks, D. J., Blanchard, S. G., Bledsoe, R. K., Chandra, G., Consler, T. G., Kliewer, S. A., et al. (1999). Bile acids: natural ligands for an orphan nuclear receptor. Science 284, 1365-1368. doi: 10.1126/science.284.5418.1365

Parséus, A., Sommer, N., Sommer, F., Caesar, R., Molinaro, A., Stahlman, M., et al. (2016). Microbiota-induced obesity requires farnesoid X receptor. Gut 66, 429-437. doi: 10.1136/gutjnl-2015-310283

Pastori, D., Carnevale, R., Nocella, C., Novo, M., Santulli, M., Cammisotto, V., et al. (2017). Gut-derived serum lipopolysaccharide is associated with enhanced risk of major adverse cardiovascular events in atrial fibrillation: effect of adherence to mediterranean diet. J. Am. Heart Assoc. 6:e005784. doi: 10.1161/JAHA.117.005784

Peng, L., He, Z., Chen, W., Holzman, I. R., and Lin, J. (2007). Effects of butyrate on intestinal barrier function in a Caco-2 cell monolayer model of intestinal barrier. Pediatr. Res. 61, 37-41. doi: 10.1203/01.pdr.0000250014.92242.f3

Plovier, H., Everard, A., Druart, C., Depommier, C., Van Hul, M., Geurts, L., et al. (2017). A purified membrane protein from Akkermansia muciniphila or the pasteurized bacterium improves metabolism in obese and diabetic mice. Nat. Med. 23, 107-113. doi: 10.1038/nm.4236

Poggi, M., Bastelica, D., Gual, P., Iglesias, M. A., Gremeaux, T., Knauf, C., et al. (2007). C3H/HeJ mice carrying a toll-like receptor 4 mutation are protected against the development of insulin resistance in white adipose tissue in response to a high-fat diet. Diabetologia 50, 1267-1276. doi: 10.1007/s00125-007-0654-8

Qiao, Y., Sun, J., Xia, S., Tang, X., Shi, Y., and Le, G. (2014). Effects of resveratrol on gut microbiota and fat storage in a mouse model with high-fat-induced obesity. Food Funct 5, 1241-1249. doi: 10.1039/c3fo60630a

Qin, J., Li, R., Raes, J., Arumugam, M., Burgdorf, K. S., Manichanh, C., et al. (2010). A human gut microbial gene catalogue established by metagenomic sequencing. Nature 464, 59-65. doi: 10.1038/nature08821

Robbins, G. R., Wen, H., and Ting, J. P. (2014). Inflammasomes and metabolic disorders: old genes in modern diseases. Mol. Cell 54, 297-308. doi: 10.1016/j.molcel.2014.03.029

Romano, K. A., Vivas, E. I., Amador-Noguez, D., and Rey, F. E. (2015). Intestinal microbiota composition modulates choline bioavailability from diet and accumulation of the proatherogenic metabolite trimethylamine- $\mathrm{N}$-oxide. MBio 6:e02481. doi: 10.1128/mBio.02481-14

Roopchand, D. E., Carmody, R. N., Kuhn, P., Moskal, K., Rojas-Silva, P., Turnbaugh, P. J., et al. (2015). Dietary Polyphenols Promote Growth of the Gut Bacterium Akkermansia muciniphila and Attenuate High-Fat Diet-Induced Metabolic Syndrome. Diabetes 64, 2847-2858. doi: 10.2337/db14-1916

Saad, M. J., Santos, A., and Prada, P. O. (2016). Linking Gut Microbiota and Inflammation to Obesity and Insulin Resistance. Physiology (Bethesda) 31, 283-293. doi: 10.1152/physiol.00041.2015

Samuel, B. S., Shaito, A., Motoike, T., Rey, F. E., Backhed, F., Manchester, J. K., et al. (2008). Effects of the gut microbiota on host adiposity are modulated by the short-chain fatty-acid binding G protein-coupled receptor, Gpr41. Proc. Natl. Acad. Sci. U.S.A. 105, 16767-16772. doi: 10.1073/pnas.0808567105

Sanchez-Tapia, M., Aguilar-Lopez, M., Perez-Cruz, C., Pichardo-Ontiveros, E., Wang, M., Donovan, S. M., et al. (2017). Nopal (Opuntia ficus indica) protects from metabolic endotoxemia by modifying gut microbiota in obese rats fed high fat/sucrose diet. Sci. Rep. 7:4716. doi: 10.1038/s41598-017-05096-4

Savage, D. C. (1977). Microbial ecology of the gastrointestinal tract. Annu. Rev. Microbiol. 31, 107-133. doi: 10.1146/annurev.mi.31.100177.000543

Sayin, S. I., Wahlstrom, A., Felin, J., Jantti, S., Marschall, H. U., Bamberg, K., et al. (2013). Gut microbiota regulates bile acid metabolism by reducing the levels of tauro-beta-muricholic acid, a naturally occurring FXR antagonist. Cell Metab. 17, 225-235. doi: 10.1016/j.cmet.2013.01.003

Schroeder, B. O., and Backhed, F. (2016). Signals from the gut microbiota to distant organs in physiology and disease. Nat Med 22, 1079-1089. doi: $10.1038 / \mathrm{nm} .4185$ 
Schugar, R. C., Shih, D. M., Warrier, M., Helsley, R. N., Burrows, A., Ferguson, D., et al. (2017). The TMAO-Producing Enzyme Flavin-Containing Monooxygenase 3 Regulates Obesity and the Beiging of White Adipose Tissue. Cell Rep. 19, 2451-2461. doi: 10.1016/j.celrep.2017.05.077

Seldin, M. M., Meng, Y., Qi, H., Zhu, W., Wang, Z., Hazen, S. L., et al. (2016). Trimethylamine N-Oxide promotes vascular inflammation through signaling of mitogen-activated protein kinase and nuclear factor-kappaB. J. Am. Heart Assoc. 5:e002767. doi: 10.1161/JAHA.115.002767

Senthong, V., Li, X. S., Hudec, T., Coughlin, J., Wu, Y., Levison, B., et al. (2016a). Plasma Trimethylamine N-Oxide, a gut microbe-generated phosphatidylcholine metabolite, is associated with atherosclerotic burden. J. Am. Coll. Cardiol. 67, 2620-2628. doi: 10.1016/j.jacc.2016.03.546

Senthong, V., Wang, Z., Fan, Y., Wu, Y., Hazen, S. L., and Tang, W. H. (2016b). Trimethylamine $\mathrm{N}$-oxide and mortality risk in patients with peripheral artery disease. J. Am. Heart Assoc. 5:e004237. doi: 10.1161/JAHA.116.004237

Senthong, V., Wang, Z., Li, X. S., Fan, Y., Wu, Y., Tang, W. H., et al. (2016c). Intestinal microbiota-generated metabolite trimethylamine-n-oxide and 5year mortality risk in stable coronary artery disease: the contributory role of intestinal microbiota in a COURAGE-like patient cohort. J. Am. Heart Assoc. 5:e002816. doi: 10.1161/JAHA.115.002816

Shang, Q., Shan, X., Cai, C., Hao, J., Li, G., and Yu, G. (2016). Dietary fucoidan modulates the gut microbiota in mice by increasing the abundance of Lactobacillus and Ruminococcaceae. Food Funct 7, 3224-3232. doi: 10.1039/C6FO00309E

Shi, H., Kokoeva, M. V., Inouye, K., Tzameli, I., Yin, H., and Flier, J. S. (2006). TLR4 links innate immunity and fatty acid-induced insulin resistance. J. Clin. Invest. 116, 3015-3025. doi: 10.1172/JCI28898

Shi, L.-L., Wang, Y., and Feng, Y. (2015). [Effect of MDG-1, a polysaccharide from Ophiopogon japonicas, on diversity of lactobacillus in diet-induced obese mice]. Zhongguo Zhong Yao Za Zhi 40, 716-721. doi: 10.4268/cjcmm20150426

Shin, N. R., Lee, J. C., Lee, H. Y., Kim, M. S., Whon, T. W., Lee, M. S., et al. (2014). An increase in the Akkermansia spp. population induced by metformin treatment improves glucose homeostasis in diet-induced obese mice. Gut 63, 727-735. doi: 10.1136/gutjnl-2012-303839

Simon, G. L., and Gorbach, S. L. (1984). Intestinal flora in health and disease. Gastroenterology 86, 174-193.

Simpson, H. L., and Campbell, B. J. (2015). Review article: dietary fibre-microbiota interactions. Aliment. Pharmacol. Ther. 42, 158-179. doi: 10.1111/apt. 13248

Singh, D. P., Singh, J., Boparai, R. K., Zhu, J., Mantri, S., Khare, P., et al. (2017). Isomalto-oligosaccharides, a prebiotic, functionally augment green tea effects against high fat diet-induced metabolic alterations via preventing gut dysbacteriosis in mice. Pharmacol. Res. 123, 103-113. doi: 10.1016/j.phrs.2017.06.015

Sonnenburg, J. L., and Backhed, F. (2016). Diet-microbiota interactions as moderators of human metabolism. Nature 535, 56-64. doi: $10.1038 /$ nature 18846

Sun, H., Ni, X., Song, X., Wen, B., Zhou, Y., Zou, F., et al. (2016). Fermented Yupingfeng polysaccharides enhance immunity by improving the foregut microflora and intestinal barrier in weaning rex rabbits. Appl. Microbiol. Biotechnol. 100, 8105-8120. doi: 10.1007/s00253-016-7619-0

Sun, X., Jiao, X., Ma, Y., Liu, Y., Zhang, L., He, Y., et al. (2016). Trimethylamine Noxide induces inflammation and endothelial dysfunction in human umbilical vein endothelial cells via activating ROS-TXNIP-NLRP3 inflammasome. Biochem. Biophys. Res. Commun. 481, 63-70. doi: 10.1016/j.bbrc.2016.11.017

Suzuki, T., Heaney, L. M., Jones, D. J., and Ng, L. L. (2017). Trimethylamine Noxide and risk stratification after acute myocardial infarction. Clin. Chem. 63, 420-428. doi: 10.1373/clinchem.2016.264853

Sweeney, T. E., and Morton, J. M. (2013). The human gut microbiome: a review of the effect of obesity and surgically induced weight loss. JAMA Surg. 148, 563-569. doi: 10.1001/jamasurg.2013.5

Tang, W. H., Wang, Z., Fan, Y., Levison, B., Hazen, J. E., Donahue, L. M., et al. (2014). Prognostic value of elevated levels of intestinal microbe-generated metabolite trimethylamine-N-oxide in patients with heart failure: refining the gut hypothesis. J. Am. Coll. Cardiol. 64, 1908-1914. doi: 10.1016/j.jacc.2014.02.617

Tang, W. H., Wang, Z., Kennedy, D. J., Wu, Y., Buffa, J. A., AgatisaBoyle, B., et al. (2015a). Gut microbiota-dependent trimethylamine N-oxide
(TMAO) pathway contributes to both development of renal insufficiency and mortality risk in chronic kidney disease. Circ. Res. 116, 448-455. doi: 10.1161/CIRCRESAHA.116.305360

Tang, W. H., Wang, Z., Levison, B. S., Koeth, R. A., Britt, E. B., Fu, X., et al. (2013). Intestinal microbial metabolism of phosphatidylcholine and cardiovascular risk. N. Engl. J. Med. 368, 1575-1584. doi: 10.1056/NEJMoa1109400

Tang, W. H., Wang, Z., Li, X. S., Fan, Y., Li, D. S., Wu, Y., et al. (2016). Increased trimethylamine $\mathrm{N}$-oxide portends high mortality risk independent of glycemic control in patients with type 2 diabetes mellitus. Clin. Chem. 63, 297-306. doi: 10.1373 /clinchem.2016.263640

Tang, W. H., Wang, Z., Shrestha, K., Borowski, A. G., Wu, Y., Troughton, R. W., et al. (2015b). Intestinal microbiota-dependent phosphatidylcholine metabolites, diastolic dysfunction, and adverse clinical outcomes in chronic systolic heart failure. J. Card. Fail 21, 91-96. doi: 10.1016/j.cardfail.2014.11.006

Thomas, C., Gioiello, A., Noriega, L., Strehle, A., Oury, J., Rizzo, G., et al. (2009). TGR5-mediated bile acid sensing controls glucose homeostasis. Cell Metab. 10, 167-177. doi: 10.1016/j.cmet.2009.08.001

Thomas, C., Pellicciari, R., Pruzanski, M., Auwerx, J., and Schoonjans, K. (2008). Targeting bile-acid signalling for metabolic diseases. Nat. Rev. Drug Discov. 7, 678-693. doi: 10.1038/nrd2619

Tolhurst, G., Heffron, H., Lam, Y. S., Parker, H. E., Habib, A. M., Diakogiannaki, E., et al. (2012). Short-chain fatty acids stimulate glucagon-like peptide-1 secretion via the G-protein-coupled receptor FFAR2. Diabetes 61, 364-371. doi: $10.2337 / \mathrm{db} 11-1019$

Trabelsi, M. S., Daoudi, M., Prawitt, J., Ducastel, S., Touche, V., Sayin, S. I., et al. (2015). Farnesoid X receptor inhibits glucagon-like peptide-1 production by enteroendocrine L cells. Nat. Commun. 6:7629. doi: 10.1038/ncomms8629

Troseid, M., Hov, J. R., Nestvold, T. K., Thoresen, H., Berge, R. K., Svardal, A., et al. (2016). Major increase in microbiota-dependent proatherogenic metabolite tMAO one year after bariatric surgery. Metab. Syndr. Relat. Disord 14, 197-201. doi: $10.1089 /$ met.2015.0120

Troseid, M., Ueland, T., Hov, J. R., Svardal, A., Gregersen, I., Dahl, C. P., et al. (2015). Microbiota-dependent metabolite trimethylamine-N-oxide is associated with disease severity and survival of patients with chronic heart failure. J. Intern. Med. 277, 717-726. doi: 10.1111/joim.12328

Turnbaugh, P. J., Ley, R. E., Hamady, M., Fraser-Liggett, C. M., Knight, R., and Gordon, J. I. (2007). The human microbiome project. Nature 449, 804-810. doi: 10.1038 /nature 06244

Vijay-Kumar, M., Aitken, J. D., Carvalho, F. A., Cullender, T. C., Mwangi, S., Srinivasan, S., et al. (2010). Metabolic syndrome and altered gut microbiota in mice lacking Toll-like receptor 5. Science 328, 228-231. doi: 10.1126/science. 1179721

Wahlstrom, A., Sayin, S. I., Marschall, H. U., and Backhed, F. (2016). Intestinal crosstalk between bile acids and microbiota and its impact on host metabolism. Cell Metab. 24, 41-50. doi: 10.1016/j.cmet.2016.05.005

Walker, J., Schueller, K., Schaefer, L. M., Pignitter, M., Esefelder, L., and Somoza, V. (2014). Resveratrol and its metabolites inhibit pro-inflammatory effects of lipopolysaccharides in U-937 macrophages in plasma-representative concentrations. Food Funct. 5, 74-84. doi: 10.1039/C3FO60236B

Wang, H., Chen, J., Hollister, K., Sowers, L. C., and Forman, B. M. (1999). Endogenous bile acids are ligands for the nuclear receptor FXR/BAR. Mol. Cell 3, 543-553. doi: 10.1016/S1097-2765(00)80348-2

Wang, J. H., Bose, S., Kim, G. C., Hong, S. U., Kim, J. H., Kim, J. E., et al. (2014). Flos Lonicera ameliorates obesity and associated endotoxemia in rats through modulation of gut permeability and intestinal microbiota. PLOS ONE 9:e86117. doi: 10.1371/journal.pone.0086117

Wang, J., Ghosh, S., and Ghosh, S. (2017). Curcumin improves intestinal barrier function: modulation of intracellular signaling, and organization of tight junctions. Am. J. Physiol. Cell Ph 312, C438-C445. doi: 10.1152/ajpcell.00235.2016

Wang, Q., Du, Z., Zhang, H., Zhao, L., Sun, J., Zheng, X., et al. (2015). Modulation of gut microbiota by polyphenols from adlay (Coix lacryma-jobi L. var. ma-yuen Stapf.) in rats fed a high-cholesterol diet. Int. J. Food Sci. Nutr. 66, 783-789. doi: 10.3109/09637486.2015.1088941

Wang, S., Huang, X. F., Zhang, P., Wang, H., Zhang, Q., Yu, S., et al. (2016). Chronic rhein treatment improves recognition memory in high-fat diet-induced obese male mice. J. Nutr. Biochem. 36, 42-50. doi: 10.1016/j.jnutbio.2016.07.008 
Wang, S., Li, Q., Zang, Y., Zhao, Y., Liu, N., Wang, Y., et al. (2017). Apple Polysaccharide inhibits microbial dysbiosis and chronic inflammation and modulates gut permeability in HFD-fed rats. Int. J. Biol. Macromol. 99, 282-292. doi: 10.1016/j.ijbiomac.2017.02.074

Wang, Y., Ames, N. P., Tun, H. M., Tosh, S. M., Jones, P. J., and Khafipour, E. (2016). High Molecular Weight Barley beta-Glucan Alters Gut Microbiota Toward Reduced Cardiovascular Disease Risk. Front. Microbiol. 7:129. doi: 10.3389/fmicb.2016.00129

Wang, Y., Shou, J. W., Li, X. Y., Zhao, Z. X., Fu, J., He, C. Y., et al. (2017). Berberine-induced bioactive metabolites of the gut microbiota improve energy metabolism. Metabolism 70, 72-84. doi: 10.1016/j.metabol.2017. 02.003

Wang, Y., Tong, J., Chang, B., Wang, B., Zhang, D., and Wang, B. (2014). Effects of alcohol on intestinal epithelial barrier permeability and expression of tight junction-associated proteins. Mol. Med. Rep. 9, 2352-2356. doi: $10.3892 / \mathrm{mmr} .2014 .2126$

Wang, Z. N., Klipfell, E., Bennett, B. J., Koeth, R., Levison, B. S., Dugar, B., et al. (2011). Gut flora metabolism of phosphatidylcholine promotes cardiovascular disease. Nature 472, 57-82. doi: 10.1038/nature09922

Wang, Z., Roberts, A. B., Buffa, J. A., Levison, B. S., Zhu, W., Org, E., et al. (2015). Non-lethal Inhibition of Gut Microbial Trimethylamine Production for the Treatment of Atherosclerosis. Cell 163, 1585-1595. doi: 10.1016/j.cell.2015.11.055

Wiest, R., Albillos, A., Trauner, M., Bajaj, J., and Jalan, R. (2017). Targeting the gut-liver axis in liver disease. J. Hepatol. 67, 1084-1103. doi: 10.1016/j.jhep.2017.05.007

Wilson, A., Mclean, C., and Kim, R. B. (2016). Trimethylamine-N-oxide: a link between the gut microbiome, bile acid metabolism, and atherosclerosis. Curr. Opin. Lipidol. 27, 148-154. doi: 10.1097/MOL.0000000000000274

Wink, M. (2015). Modes of action of herbal medicines and plant secondary metabolites. Medicines 2:251. doi: 10.3390/medicines2030251

Woodside, J. V., Young, I. S., and Mckinley, M. C. (2013). Fruit and vegetable intake and risk of cardiovascular disease. Proc. Nutr. Soc. 72, 399-406. doi: 10.1017/S0029665113003029

Woting, A., and Blaut, M. (2016). The intestinal microbiota in metabolic disease. Nutrients 8:202. doi: 10.3390/nu8040202

Wu, W., Sun, M., Chen, F., Cao, A. T., Liu, H., Zhao, Y., et al. (2016). Microbiota metabolite short-chain fatty acid acetate promotes intestinal IgA response to microbiota which is mediated by GPR43. Mucosal Immunol. 10, 946-956. doi: $10.1038 / \mathrm{mi} .2016 .114$

Xiao, M., Yang, J., Feng, Y., Zhu, Y., Chai, X., and Wang, Y. (2017). Metaproteomic strategies and applications for gut microbial research. Appl. Microbiol. Biotechnol. 101, 3077-3088. doi: 10.1007/s00253-017-8215-7

Xie, W., Gu, D., Li, J., Cui, K., and Zhang, Y. (2011). Effects and action mechanisms of berberine and Rhizoma coptidis on gut microbes and obesity in high-fat diet-fed C57BL/6J mice. PLOS ONE 6:e24520. doi: 10.1371/journal.pone.00 24520

Xu, J. H., Liu, X. Z., Pan, W., and Zou, D. J. (2017). Berberine protects against dietinduced obesity through regulating metabolic endotoxemia and gut hormone levels. Mol. Med. Rep. 15, 2765-2787. doi: 10.3892/mmr.2017.6321

$\mathrm{Xu}$, J., Chen, H. B., and Li, S. L. (2017). Understanding the molecular mechanisms of the interplay between herbal medicines and gut microbiota. Med. Res. Rev. 37, 1140-1185. doi: 10.1002/med.21431

Xu, J., Lian, F., Zhao, L., Zhao, Y., Chen, X., Zhang, X., et al. (2015). Structural modulation of gut microbiota during alleviation of type 2 diabetes with a Chinese herbal formula. ISME J. 9, 552-562. doi: 10.1038/ismej.2014.177

Xu, K. Y., Xia, G. H., Lu, J. Q., Chen, M. X., Zhen, X., Wang, S., et al. (2017). Impaired renal function and dysbiosis of gut microbiota contribute to increased
trimethylamine-N-oxide in chronic kidney disease patients. Sci. Rep. 7:1445 doi: 10.1038/s41598-017-01387-y

Xu, P., Wang, J., Hong, F., Wang, S., Jin, X., Xue, T., et al. (2017). Melatonin prevents obesity through modulation of gut microbiota in mice. J. Pineal. Res. 62:e12399. doi: 10.1111/jpi.12399

Yan, H., Lu, J., Wang, Y., Gu, W., Yang, X., and Yu, J. (2017). Intake of total saponins and polysaccharides from Polygonatum kingianum affects the gut microbiota in diabetic rats. Phytomedicine 26, 45-54. doi: 10.1016/j.phymed.2017.01.007

Yang, Y., Chen, G., Yang, Q., Ye, J., Cai, X., Tsering, P., et al. (2017). Gut microbiota drives the attenuation of dextran sulphate sodium-induced colitis by Huangqin decoction. Oncotarget. 8, 48863-48874. doi: 10.18632/oncotarget.16458

Young, R. P., Hopkins, R. J., and Marsland, B. (2016). The Gut-Liver-Lung Axis. Modulation of the Innate Immune Response and Its Possible Role in Chronic Obstructive Pulmonary Disease. Am. J. Respir. Cell Mol. Biol. 54, 161-169. doi: 10.1165/rcmb.2015-0250PS

Yu, H., Guo, Z., Shen, S., and Shan, W. (2016). Effects of taurine on gut microbiota and metabolism in mice. Amino Acids 48, 1601-1617. doi: 10.1007/s00726-016-2219-y

Zhang, X. H., Huang, B., Choi, S. K., and Seo, J. S. (2012b). Anti-obesity effect of resveratrol-amplified grape skin extracts on 3T3-L1 adipocytes differentiation. Nutr. Res. Pract. 6, 286-293. doi: 10.4162/nrp.2012.6.4.286

Zhang, X., Zhao, Y., Xu, J., Xue, Z., Zhang, M., Pang, X., et al. (2015). Modulation of gut microbiota by berberine and metformin during the treatment of high-fat diet-induced obesity in rats. Sci. Rep. 5:14405. doi: 10.1038/srep14405

Zhang, X., Zhao, Y., Zhang, M., Pang, X., Xu, J., Kang, C., et al. (2012a). Structural changes of gut microbiota during berberine-mediated prevention of obesity and insulin resistance in high-fat diet-fed rats. PLoS ONE 7:e42529. doi: 10.1371/journal.pone.0042529

Zhou, J., Ma, Y. H., Zhou, Z., Chen, Y., Wang, Y., and Gao, X. (2015). Intestinal absorption and metabolism of epimedium flavonoids in osteoporosis rats. Drug Metab. Dispos. 43, 1590-1600. doi: 10.1124/dmd.115.064386

Zhou, S. S., Xu, J., Zhu, H., Wu, J., Xu, J. D., Yan, R., et al. (2016). Gut microbiota-involved mechanisms in enhancing systemic exposure of ginsenosides by coexisting polysaccharides in ginseng decoction. Sci. Rep. 6:22474. doi: $10.1038 /$ srep 22474

Zhu, W., Gregory, J. C., Org, E., Buffa, J. A., Gupta, N., Wang, Z., et al. (2016). Gut Microbial Metabolite TMAO Enhances Platelet Hyperreactivity and Thrombosis Risk. Cell 165, 111-124. doi: 10.1016/j.cell.2016.02.011

Zhu, W., Wang, Z., Tang, W. H. W., and Hazen, S. L. (2017). Gut Microbe-Generated Trimethylamine N-Oxide From Dietary Choline Is Prothrombotic in Subjects. Circulation 135, 1671-1673. doi: 10.1161/CIRCULATIONAHA.116.025338

Zhu, Y., Jameson, E., Crosatti, M., Schafer, H., Rajakumar, K., Bugg, T. D., et al. (2014). Carnitine metabolism to trimethylamine by an unusual Riesketype oxygenase from human microbiota. Proc. Natl. Acad. Sci. U.S.A. 111, 4268-4273. doi: 10.1073/pnas.1316569111

Conflict of Interest Statement: The authors declare that the research was conducted in the absence of any commercial or financial relationships that could be construed as a potential conflict of interest.

Copyright (c) 2017 Lyu, Wang, Fan, Wang, Xu and Zhu. This is an open-access article distributed under the terms of the Creative Commons Attribution License (CC BY). The use, distribution or reproduction in other forums is permitted, provided the original author(s) or licensor are credited and that the original publication in this journal is cited, in accordance with accepted academic practice. No use, distribution or reproduction is permitted which does not comply with these terms. 